\title{
Silicon Photonic Micro-Ring Resonators for Chemical and Biological Sensing: A Tutorial
}

\author{
Patrick Steglich, Member, IEEE, Dominik G. Rabus, Senior Member, IEEE, Cinzia Sada, Martin Paul, \\ Michael G. Weller, Christian Mai, and Andreas Mai, Member, IEEE
}

\begin{abstract}
Silicon photonic micro-ring resonators (MRR) developed on the silicon-on-insulator (SOI) platform, owing to their high sensitivity and small footprint, show great potential for many chemical and biological sensing applications such as label-free detection in environmental monitoring, biomedical engineering, and food analysis. In this tutorial, we provide the theoretical background and give design guidelines for SOI-based MRR as well as examples of surface functionalization procedures for labelfree detection of molecules. After introducing the advantages and perspectives of MRR, fundamentals of MRR are described in detail, followed by an introduction to the fabrication methods, which are based on a complementary metal-oxide semiconductor (CMOS) technology. Optimization of MRR for chemical and biological sensing is provided, with special emphasis on the optimization of waveguide geometry. At this point, the difference between chemical bulk sensing and label-free surface sensing is explained, and definitions like waveguide sensitivity, ring sensitivity, overall sensitivity as well as the limit of detection (LoD) of MRR are introduced. Further, we show and explain chemical bulk sensing of sodium chloride $(\mathrm{NaCl})$ in water and provide a recipe for label-free surface sensing.
\end{abstract}

Index Terms-Biosensors, Biophotonics, Chemical and biological sensors, Microresonators, Nanophotonics, Optical sensors, Optoelectronic and photonic sensors, Ring resonators, Silicon photonics.

\section{INTRODUCTION}

$\mathbf{I}$ NTEGRATED photonic sensors give perspectives to a multitude of chemical and biological sensing applications such as food analysis [1], [2], virus detection [3], healthcare [4], [5], and environmental monitoring [6]. The term integrated photonic sensor refers to optical sensors, which are typically composed of either interferometers or resonators, realized in a silicon-based photonic integrated circuit (PIC). PICs can be compared to electronic integrated circuits with the difference that they use photons instead of electrons to transport information. As opposed to electronic integrated circuits, which are typically constructed of transistor arrays, PICs employ a

Patrick Steglich and Andreas Mai are with the IHP - Leibniz-Institut für innovative Mikroelektronik, 15236 Frankfurt (Oder), Germany, and also with the Technische Hochschule Wildau, 15745 Wildau, Germany (e-mail: steglich@ihp-microelectronics.com,mai@ihp-microelectronics.com).

Dominik G. Rabus is with Reutlingen University, Alteburgstr. 150, 72762 Reutlingen, Germany (e-mail: dominik.rabus@ reutlingen-university.de).

Cinzia Sada is with University of Padova, Department of Physics and Astronomy Galileo Galilei, Padova, Italy (e-mail: cinzia.sada@unipd.it).

Martin Paul and Michael G. Weller are with the Federal Institute for Materials Research and Testing (BAM), 12489 Berlin, Germany (e-mail: martin.paul@bam.de, michael.weller@bam.de).

Christian Mai is with the IHP - Leibniz-Institut für innovative Mikroelektronik, 15236 Frankfurt (Oder), Germany (e-mail: cmai@ihpmicroelectronics.com).

Manuscript received January 29, 2021; revised ??, 2021. range of components like optical waveguides and gratings to guide, separate, polarize, modulate, couple, and, ultimately, to generate and detect light [7], [8]. The underlying technology is using porous silicon [9], [10], silicon nitride [11] or siliconon-insulator (SOI) wafer with crystalline silicon [12] as basis material for the sensor device. This tutorial is focusing on SOI-based PICs. This is motivated by the mature fabrication processes offered by open-access pilot lines, making SOIbased PICs attractive for both, science and industry [13]. Besides that, SOI-based PICs can be jointly integrated with electronics, which is known as electronic-photonic integrated circuits (EPIC) [14].

Like many types of chip-integrated biosensors, photonic biosensors can provide various advantages. For example, they are resistant against electromagnetic interference, exhibit high sensitivity and low detection limit, while they provide an ultrasmall footprints, allow labeled and label-free sensing as well as multiplexing of several analytes at the same time. Furthermore, integration into lab-on-a-chip systems for rapid sample-toanswer capabilities is feasible.

Due to its small footprint, i.e. the area occupied on the chip, and high sensitivity, optical resonators are widely employed. In particular, micro-ring resonators (MRR) are the most important type since they provide excellent sensitivity and have been intensively studied over the last two decades for the label-free detection of biomarkers [15]. Since MRRs exhibit a small footprint, they are widely used by scientists. This is motivated by the fact that the fabrication costs scales with chip-size. Beside that, they can be fabricated in pilot lines using photolithography, which makes them attractive for mass production. MRR-based biosensors are widely used and are investigated since two decades. A comprehensive literature review can be found in [12], [15], [16].

The working principle is as follows. Light is coupled to the MRR from a straight waveguide via evanescence field coupling, as indicated in Figure 1. The light is then circulating inside the ring if the resonance condition is fulfilled. In this case, resonance peaks appear in the optical spectrum, as shown in Figure 2, if the wavelength of the light matches a whole number of times inside the optical length of the ring, leading to the condition

$$
L n_{e f f}=m \lambda_{r e s}
$$

where $L$ represents the circumference of the ring of radius $r$ measured from the center of the ring to the center of the waveguide, $n_{e f f}$ is the effective refractive index of the ringwaveguide, $m$ is a whole number, also called as mode number 


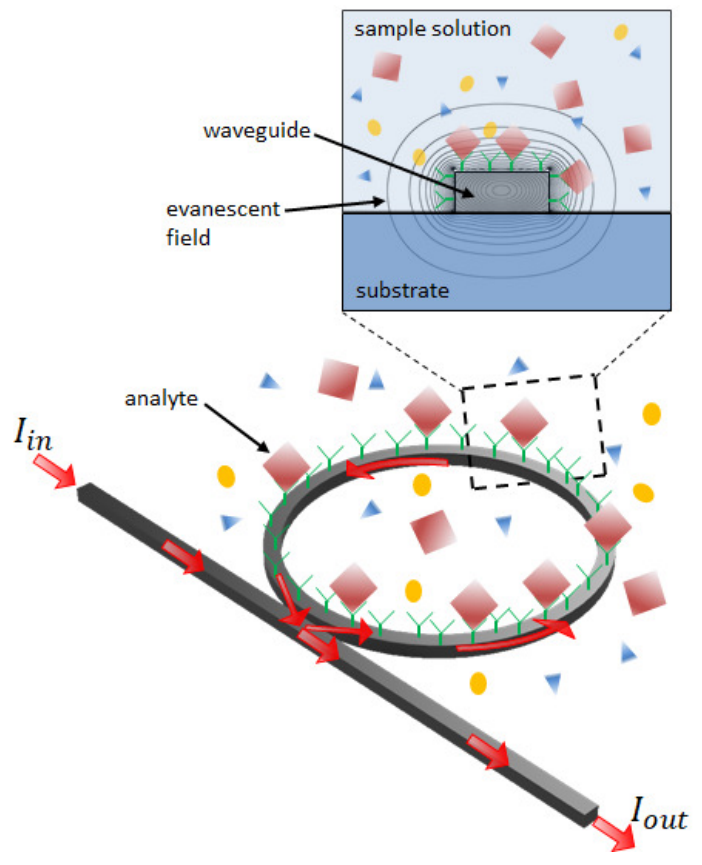

Figure 1. Simplified schematic of a silicon photonic micro-ring sensor. (a)

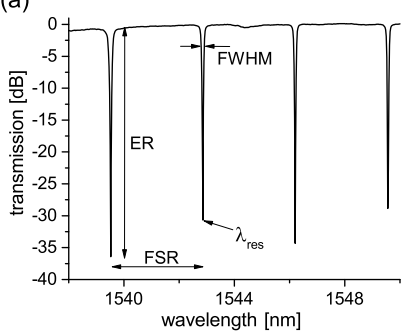

(b)

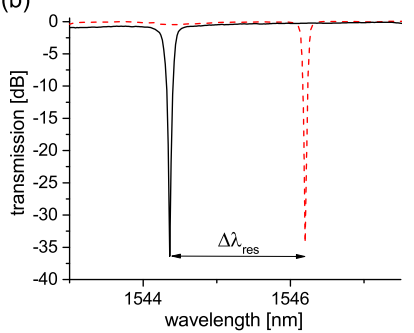

Figure 2. a) Transmission spectrum of a MRR. b) Resonance wavelength shift due to a change of the cladding refractive index. ER = Extinction Ration; FSR $=$ Free Spectral Range; FWHM = Full Width at Half Maximum; $\lambda_{r e s}$ = resonance wavelength; $\Delta \lambda_{\text {res }}=$ resonance wavelength shift

( $m=1,2,3, \ldots)$, and $\lambda_{r e s}$ is the wavelength at which the resonance occurs. Please note that the shape of the ring is not necessarily a perfect circle. For example, to increase the interaction length, i.e. the total circumference, racetrack [17] or spiral [18] shapes are used. The effective refractive index of the ring-waveguide takes the geometry of the waveguide and the refractive index of all materials into account, i.e. the core (silicon), the substrate and the cladding material. The reason for that is, that the light is not $100 \%$ confined inside the waveguide core. The evanescence field, which represents the portion of light that is penetrating inside the surrounding material, can interact with the fluid. Thus, a weak perturbation to the evanescent field leads to a change of the effective refractive index and, consequently, to a change of the resonance condition. This perturbation can be provoked by a small change of the refractive index of the fluid. For example, if the salt concentration in water is changing, the refractive index and consequently the resonance condition is changed, too. This is known as chemical bulk sensing because the medium refractive index near to the waveguide is measured. To enable a specific detection of molecules, e.g. label-free detection of biomarkers, the surface of the MRR is functionalized with an adsorbed layer [19]. In this way, specific molecules are bound on the surface, inducing a resonance shift. This approach is known as label-free surface sensing. The sensing information, i.e. the resonance shift, can be quantitatively interrogated by measuring either the resonance peak shift or the intensity change a certain wavelength.

By using complementary metal-oxide semiconductor (CMOS) processes, the fabrication of silicon photonic sensors can be done in a similar way as electronic integrated circuits are fabricated. In this scenario, MRR-based sensors can be fabricated and post-processed with mature CMOS technologies, which opens the door for a scalable massproduction. After chip fabrication, the surface of the sensor can be functionalized with ligands for a specific detection of target molecules. Microfluidics are mostly used to control the flow rate and to reduce the required measurement time [20]. At this point, it becomes clear that working with MRR-based photonic sensors requires an interdisciplinary background. This tutorial is intended to provide the required background.

The following list provides a better understanding of some characteristics and properties of silicon photonic MRR-based biosensors:

(a) Mass-production

Using CMOS-based fabrication flows, photonic sensor chips are ready to be produced in a large-scale.

(b) Rapid results

Silicon photonic sensors based on MRRs have been proven to provide measurement results within a few minutes and can be used as quick test solution.

(c) Miniaturization

Photonic chips in a CMOS technology are typically 3 to $30 \mathrm{~mm}^{2}$ in size. MRR-based photonic sensors allow the realization of 5 to 30 sensors on such an area due to their small footprint.

(d) Sustainable

The small sensor size reduces the amount of chemicals required for the surface functionalization as well as the amount of the required sample (blood, water, etc.). For instance, simply dropping the sample on the sensor surface can be enough [21].

(e) Multiplexing

The simultaneous measurement of different substances can be simply realized when several MRR are placed on one chip, while each MRR is selectively functionalized [22].

\section{(f) Digital analysis}

The optical signal is directly translated into an electrical signal by using a chip-integrated photodiode for each MRR. In this way, the data can be digitally analyzed and data can be transferred secure and fast.

This tutorial aims to provide an introduction to the basic concepts of MRR in section II and fabrication methodologies in section III. A guidance to design optimized MRR for sensing applications is provided in section IV. Besides that, an introduction to the chemical surface functionalization is 
(a)

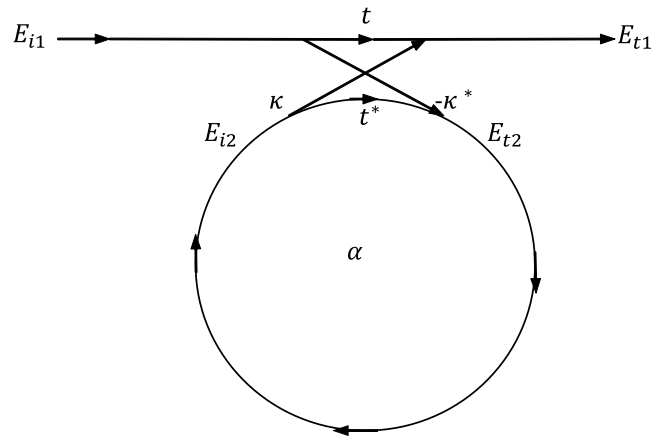

(b)

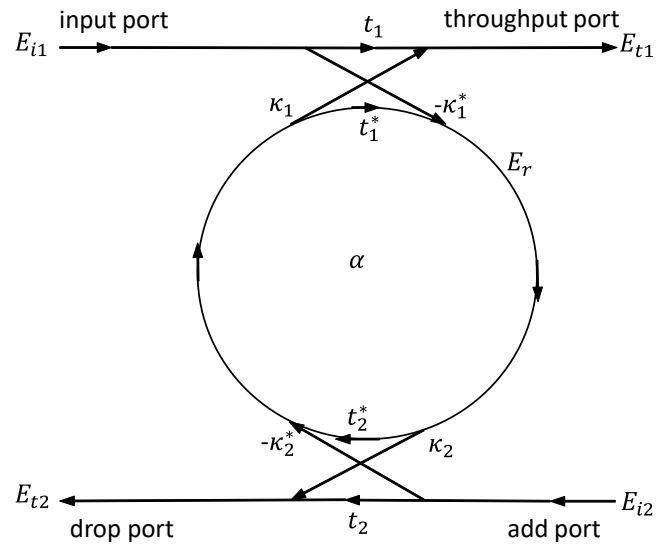

Figure 3. Model of a single ring resonator with: (a) one single coupled waveguide; (b) two coupled waveguides, the four ports of the ring resonator are referred to as input port, throughput port, drop port and add port, respectively.

given in section V. Finally, different experimental set-ups are described and discussed in section VI.

\section{Fundamentals of Micro-Ring Resonators}

The basic configuration of a MRR consists of unidirectional coupling between a ring with radius $r$ and one or two waveguides [23]. By defining $E$ as the complex electric field amplitude of the propagating mode, the overall behavior and response of a MRR can be easily described in terms of the so called Transfer Matrix where input (subscript $i$ ) and transmitted (subscript $t$ ) fields are conjugated by a matrix, containing the physical effects responsible for the coupling process between the waveguide and the ring propagating modes, respectively. The system can be solved in an analytical way in case of excitation of a single MRR unidirectional mode, zero loss coupling, single polarization and none of the waveguide segments and coupler elements couple waves of different polarization. Under these conditions, losses occurring along the propagation of light in the MRR filter can be included in the attenuation constant and the occurring interaction can be described by the simplified matrix relation. In general, it can be simplified in building blocks made of one or two coupled waveguides respectively, independently of the final overall complexity of the system under study.
In the case of one waveguide, as shown in Figure 3a, it can be demonstrated that [24]

$$
\left(\begin{array}{l}
E_{t 1} \\
E_{t 2}
\end{array}\right)=\left(\begin{array}{cc}
t & \kappa \\
-\kappa^{*} & t^{*}
\end{array}\right)\left(\begin{array}{l}
E_{i 1} \\
E_{i 2}
\end{array}\right),
$$

where $t$ indicates the coupling losses and $\kappa$ the coupling parameter, both depending on the specific coupling mechanism used. The complex mode amplitudes $E$ are normalized so that their squared magnitude corresponds to the modal power and $*$ denotes the conjugated complex value of $t$ and $\kappa$ respectively. The matrix is symmetric because the networks under consideration are reciprocal, which leads to the relation:

$$
|\kappa|^{2}+|t|^{2}=1
$$

In order to further simplify the model, $E_{i 1}$ is often put to be equal to 1 so that the round trip in the ring is given by

$$
E_{i 2}=\alpha e^{j \theta} E_{t 2},
$$

where $\alpha$ is the loss coefficient of the ring (zero loss: $\alpha=1$ ) and

$$
\theta=\omega \frac{L}{c}=4 \pi^{2} n_{\text {eff }} \frac{r}{\lambda} .
$$

Here, $c$ is the phase velocity of the ring mode $\left(c=c_{0} / n_{\text {eff }}\right)$ and the fixed angular frequency $\omega=k c_{0}$, where $c_{0}$ refers to the vacuum speed of light, vacuum wavenumber $k=2 \pi / \lambda$, wavelength in vacuum $\lambda$ and, for completeness, $\beta=k n_{\text {eff }}$ is the propagation constant. The transmission power $P_{t 1}$ in the output waveguide, is therefore given by

$$
P_{t 1}=\left|E_{t 1}\right|^{2}=\frac{\alpha^{2}+|t|^{2}+\alpha|t| \cos \left(\theta+\phi_{t}\right)}{1+\alpha^{2}|t|^{2}-2 \alpha|t| \cos \left(\theta+\phi_{t}\right)}
$$

and the absolute module of coupling losses is then

$$
t=|t| e^{\left(j \phi_{t}\right)}
$$

where $\phi_{t}$ represents the phase of the coupler. The circulating power $P_{i 2}$ in the ring is given by

$$
P_{i 2}=\left|E_{i 2}\right|^{2}=\frac{\alpha^{2}\left(1-|t|^{2}\right)}{1+\alpha^{2}|t|^{2}-2 \alpha|t| \cos \left(\theta+\phi_{t}\right)} .
$$

As a consequence, since at resonance $\left(\theta+\phi_{t}\right)=2 \pi m$ where $m$ is an integer, the following equation for the transmission power at resonance is obtained:

$$
P_{t 1, \text { res }}=\left|E_{t 1, \text { res }}\right|^{2}=\frac{(\alpha-|t|)^{2}}{(1-\alpha|t|)^{2}}
$$

and

$$
P_{i 2, \text { res }}=\left|E_{i 2, \text { res }}\right|^{2}=\frac{\alpha^{2}\left(1-|t|^{2}\right)}{(1-\alpha|t|)^{2}},
$$

for the circulating power at resonance. It is worth mentioning that when $\alpha=|t|$ (i.e. the internal losses are equal to the coupling losses), the transmitted power becomes 0 corresponding to destructive interference (known in literature as critical coupling).

The second basic MRR system is the so-called add-drop configuration, consisting of one input, one output waveguide and the ring resonator, as shown in Figure $3 b$. The values of $E_{t 1}$ and $E_{t 2}$ can be calculated in an analytical way with a similar approach than the one used for a single coupled 


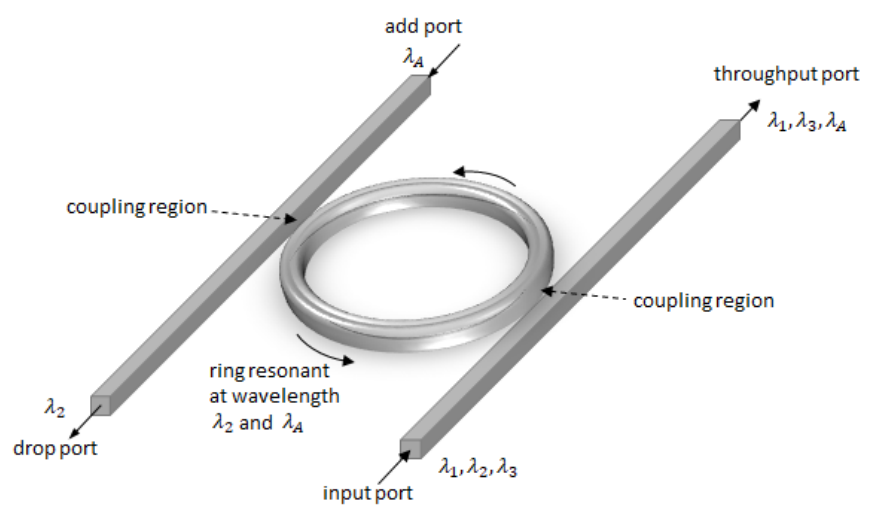

Figure 4. Ring resonator channel dropping filter.

waveguide (for further details see [24]). In particular, if $E_{i 1}$ is defined equal to 1 as before, it can be derived that at resonance, the output power from the drop port is given by:

$$
P_{t 2, \text { res }}=\left|E_{t 2, \text { res }}\right|^{2}=\frac{\left(1-\left|t_{1}\right|^{2}\right)\left(1-\left|t_{2}\right|^{2}\right) \alpha}{\left(1-\alpha\left|t_{1} t_{2}\right|\right)^{2}}
$$

The throughput port mode amplitude $E_{t 1}$ is zero at resonance for identical symmetrical couplers $t_{1}=t_{2}$ if $\alpha=1$. This condition corresponds to the case when the wavelength on resonance is fully extracted by the MRR. The value of $\alpha=1$ can only be achieved by the implementation of gain incorporated in the MRR to compensate the waveguide losses. The value of the loss coefficient $\alpha$ is fixed in a purely passive MRR. A possibility of achieving minimum intensity $\left(P_{t 1}=0\right)$ at resonance of the output transmission $P_{t 1}$ at the throughput port is to adjust the coupling parameters $t_{1}, t_{2}$ to the loss coefficient $\alpha$, which is

$$
\alpha=\left|\frac{t_{1}}{t_{2}}\right| .
$$

If the MRR is lossless $(\alpha=1)$, then the couplers have to be symmetric in order to achieve minimum intensity.

A standard application for a MRR is an integrated optical filter, which can be advantageously used to use a broadband light source instead of an expensive tunable laser [25]. In this case the two straight waveguides, also known as the bus or the port waveguides respectively, are coupled either by directional couplers through the evanescent field or by multimode interference couplers to the MRR. A simpler configuration is obtained, when the second bus or port waveguide is removed. Figure 4 shows a sketch of the filter working principle in the twowaveguide geometry for a MRR at $\lambda_{A}, \lambda_{2}$ The wavelengths $\lambda_{1}, \lambda_{2}$, and $\lambda_{3}$ are injected in the input port but, by way of the ring coupling at the coupling regions, only $\lambda_{2}$ is dropped off at the drop port whilst $\lambda_{1}$ and $\lambda_{3}$ are transmitted to the throughput port together with $\lambda_{A}$ In fact, $\lambda_{A}$ being injected by way of the add port, is coupled by the ring to reach the throughput port.

Ring resonator performances are described in terms of figures of merit that fully represent its response [24]:

1) Free Spectral Range (FSR), i.e the distance between resonance peaks $\left(\left|\lambda_{\text {res }, 1}-\lambda_{\text {res }, 2}\right|\right)$. The $F S R$ describes the difference between the vacuum wavelengths corresponding to two resonant conditions. If $n_{\text {eff }}$ is weakly dependent on $\lambda$, the FSR can be calculated by:

$$
F S R=-\frac{2 \pi}{L}\left(\frac{\delta \beta}{\delta \lambda}\right)^{-1} \approx \frac{\lambda^{2}}{n_{e f f} L}
$$

Otherwise, if the wavelength dependence of the effective index can not be neglected, the group refractive index can be used instead of the effective index whenever appropriate avoiding the approximation and obtaining more accurate values. In this case the modified $F S R$ is then given by:

$$
F S R=\frac{\lambda^{2}}{n_{g} L}
$$

2) Full Width at Half Maximum (FWHM), also known as 3$\mathrm{dB}$ bandwidth of the resonance line-shape. Assuming that the coupling coefficients are real, loss-less and without a phase-term, and if the loss in the ring is negligible and the coupling is symmetric $\left(t=t_{1}=t_{2}\right)$ in the wavelength domain, it results that:

$$
F W H M=\frac{\lambda^{2}}{\pi n_{e f f} L} \frac{1-t^{2}}{t}
$$

In case of weak coupling and $\lambda \gg \Delta \lambda$ :

$$
F W H M=\frac{\kappa^{2} \lambda^{2}}{\pi n_{e f f} L}
$$

3) Finesse $(F)$, defined as the ratio of the $F S R$ and the line-width of a resonance for a specific wavelength $(F W H M)$ :

$$
F=\frac{F S R}{F W H M}=\pi \frac{t}{1-t^{2}}
$$

However, if $\kappa \ll 1$ it can be shown that:

$$
F \approx \frac{\pi}{\kappa^{2}}
$$

4) Quality factor $Q$, which is a measure of the sharpness of the resonance. It is defined as the ratio of the operation wavelength and the resonance width:

$$
Q=\frac{\lambda_{\text {res }}}{F W H M}=\pi \frac{n_{e f f} L}{\lambda} \frac{t}{1-t^{2}}=\frac{n_{e f f} L}{\lambda} F
$$

The quality factor can also be regarded as the stored energy divided by the power lost per optical cycle.

5) Intensity enhancement or buildup factor $B$, The intensity in the ring resonator can be much higher than that in the bus waveguides, as the traveling wave in the ring resonator interferes constructively at resonance with the input wave and thus the amplitude builds up. In addition to this intensity increase, the field also experiences a phase-shift of an integral multiple of $2 \pi$ in one round trip. The intensity enhancement or buildup factor $B$ represents this increase and for single coupled waveguide (Figure 3a) it is given by:

$$
B=\left|\frac{E_{i 2}}{E_{i 1}}\right|=\left|\frac{-\alpha \kappa^{*}}{-\alpha t^{*}+e^{-j \theta}}\right|^{2}
$$


Whilst for a two coupled waveguide (Figure 3b) the buildup factor is given by:

$$
B=\left|\frac{E_{r}}{E_{i 1}}\right|=\left|\frac{-\kappa_{1}^{*}}{1-t_{1}^{*} t_{2}^{*} \alpha e^{j \theta}}\right|^{2}
$$

On resonance, the intensity enhancement factor becomes:

$$
B=\left|\frac{-\kappa_{1}^{*}}{1-t_{1}^{*} t_{2}^{*} \alpha}\right|^{2}
$$

Please note that $B$ tends to $F / \pi$ for a lossless resonator and setting $\kappa_{1}=\kappa_{2}=\kappa$ which is $\ll 1$. For the allpass configuration and on resonance, the buildup factor is given by

$$
B=\frac{1+t}{1-t},
$$

where $t_{1}=t_{2}=t$.

6) Optical losses, an important factor for the MRR performance. They are often included by insertion of the parameter $\alpha$ and can originate either from light scattering, material absorption or bending losses. Light scattering and the material absorption have been widely investigated in straight waveguides and easily taken care of. In case of MRR, bending losses $\alpha_{b e n d}$ have to be included and scale with the curvature radius $r$ with a complex expression. However, semi-empirical relations have been reported [24]:

$$
\begin{aligned}
\left.\alpha_{\text {bend }}\right|_{\text {planar }} & =-\ln \left(\frac{P_{\text {rad }}}{P_{\text {inc }}}\right) \\
& \propto \alpha_{0} e^{-\frac{2}{3} \frac{2 \pi}{\lambda} r \frac{\sqrt[3]{n_{c}^{2}-n_{s}^{2}}}{n_{c}^{2}}}
\end{aligned}
$$

and

$$
\left.\alpha_{\text {bend }}\right|_{\text {channel }} \propto 0.72 \frac{4 \pi}{\lambda}\left(n_{c}-n_{s}\right) 10^{-\frac{1}{7.6} \frac{2 \pi}{\lambda} r n_{s}} \frac{\sqrt[3]{n_{c}^{2}-n_{s}^{2}}}{n_{c}^{2}}
$$

Here, $P_{\text {rad }}$ is the radiated power due to the bend, $P_{i n c}$ is the incident power, and $\alpha_{0}$ is a constant depending on the waveguide thickness. It clearly emerges that low losses can be achieved by higher refractive index contrast $\Delta n$ and larger curvature radius $r$.

7) Time dependent response. The understanding of the dynamic response or also called time dependent response of MRRs is necessary, e.g., for kinetic exclusion analysis. Here we discuss theoretical aspects for a better understanding of the physical background. If the ring supports a traveling wave of amplitude $E(t), P(t)$ represents the total power flowing through any cross-section of the ring waveguide at time $t$. The ring is then regarded as an oscillator of energy amplitude $e(t)$, normalized so that $p(t)$ represents the total energy stored in the ring. In this case it results that

$$
p(t)=|e(t)|^{2}=P(t) \frac{2 \pi r}{v_{g}},
$$

where $v_{g}$ is the group velocity. The MRR presents a resonant frequency of $\omega_{\text {res }}$ and amplitude decay timeconstant of $1 / \tau$. The decay rate includes the contribution of the power coupled to the transmitted wave $\left(1 / \tau_{i e}\right)$, the power lost due to intrinsic effects $\left(1 / \tau_{t r}\right)$, and power coupled to the output waveguide $\left(1 / \tau_{t 2}\right)$ leading to:

$$
\frac{1}{\tau}=\frac{1}{\tau_{t r}}+\frac{1}{\tau_{i e}}+\frac{1}{\tau_{t 2}}
$$

The time rate of change in ring energy can then be written as:

$$
\frac{d}{d t} e=\left(j \omega_{\text {res }}-\frac{1}{\tau}\right) e-\kappa^{*} E_{i 1}
$$

Please note, the relation between the coupling parameter $\kappa$ and the decay rates of the transmitted wave $1 / \tau_{t r}$ and the output waveguide $1 / \tau_{t 2}$, is determined by power conservation.

It is worth mentioning that another approach to simulate ring resonator filters is by using the Z-transformation to describe the spectral and temporal response of ring resonator filters, described in detail in [26]. The Z-transform is an analytic extension of the discrete-time Fourier transform (DTFT) which converts a discrete time signal into a complex-variable frequency signal:

$$
H(z)=\sum_{n=-\infty}^{\infty} h(n) z^{-n}
$$

$Z$ is a complex variable and $h(n)$ is the impulse response of a filter or the values of a discrete signal. Each term $z^{-n}$ represents a delay. Of particular interest is when the absolute value of $|z|=1$. This is called the unit circle in the complex plane where pole and zero locations of the function $H(z)$, which is evaluated along $z=\exp (j \omega)$, are plotted. A ring resonator has a response which can be expressed in the form:

$$
H(z)=\sum_{n=-\infty}^{\infty} a^{n} z^{-n}=\frac{1}{1-a z^{-1}}
$$

In the case of ring resonator filters, $|z|=1$ corresponds to resonant frequencies. A complete roundtrip of the unit circle corresponds to the FSR of the filter. Poles and zeros are related to the filters frequency spectrum by their position on the complex plane. A zero positioned on the unit circle results in zero transmission at the frequency corresponding to the angle of this zero. A pole on the other hand on the unit circle causes unity transmission at the corresponding frequency. As poles and zeros move away from the unit circle, their effect on the magnitude spectrum is reduced. The basic configuration of an add-drop ring resonator filter (Figure $3 b$ ) is the simplest filter with a single pole response and by using the $Z$-transform approach, it can be represented as in Figure 5. The basic configurations previously described are the building block of complex system such as serially coupled configuration and parallel coupled configuration respectively (see Figure 6). In the serially coupled configuration, each ring resonator is coupled to one another so that the signal is dropped from the input port to the drop port, passing sequentially through each resonator (Figure 6a). This configuration is characterized by sequential power transfer and, consequently, all resonators must be precisely resonant at a common wavelength. The resulting resonant line shape in the series configuration is therefore determined physically by the separations between 

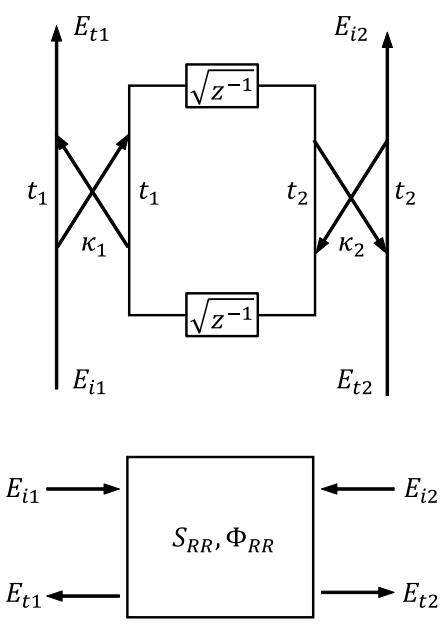

Figure 5. $Z$-transform layout of an add-drop ring resonator filter.

the ring resonators. In this case, the FSR of the double ring resonators coupled in series with two different radii is expressed by

$$
F S R=m_{1} F S R_{1}=m_{2} F S R_{2},
$$

which leads to

$$
F S R=\left|m_{2}-m_{1}\right| \frac{F S R_{1} F S R_{2}}{\left|F S R_{1}-F S R_{2}\right|},
$$

where $m_{1}$ and $m_{2}$ are coprime numbers. As a consequence, the FSR as evaluated from Eq. 32 is significantly larger than the FSR of a single MRR. Therefore, it is also called extended FSR and the underlying effect is called Vernier effect. The Vernier effect causes the transmission peaks of the ring resonators within the overall obtained FSR to be suppressed, which results in the larger $F S R$ than would be achieved for a single MRR. A figure of merit that is used to estimate the performance of a Vernier-based MRR is the Vernier gain:

$$
G=\frac{F S R_{1}}{\left|F S R_{1}-F S R_{2}\right|}
$$

Silicon photonic sensors based on the Vernier effect have shown an enhanced sensitivity compared to single MRR [27]. However, it should be noticed that the design of Vernier-based MRRs is challenging because the position of the resonances depends drastically on fabrication tolerances [28].

In the parallel-coupled configuration, instead, all resonators are coupled to both the input and drop port waveguides, without the requirement that must be coupled directly to one another (Figure 6b). An optical signal in the parallel configuration passes through all ring resonators simultaneously. The resonators are indirectly coupled to each other by the optical path lengths along the input and output waveguides that interconnect the MRRs and these lengths determine the resonant line shapes. The overall FSR can be increased by adjusting the length of the waveguide joining the two ring resonators. It is worth mentioning that the parallel configuration can be treated as a grating. In this case constructive interference of the reflected waves from each ring resonator is (a)

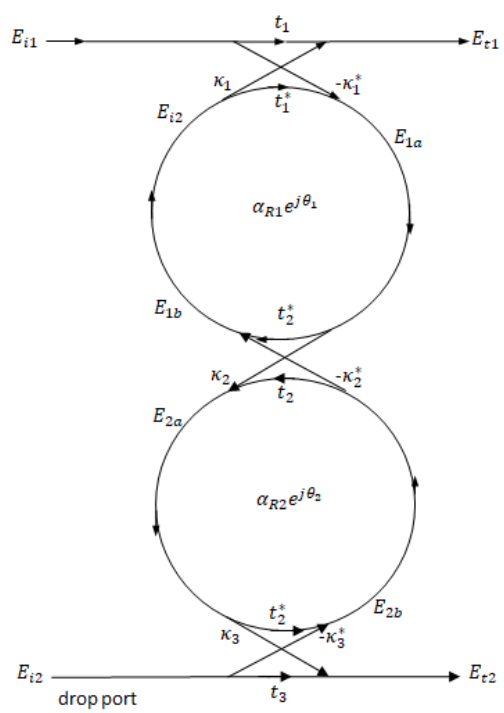

(b)

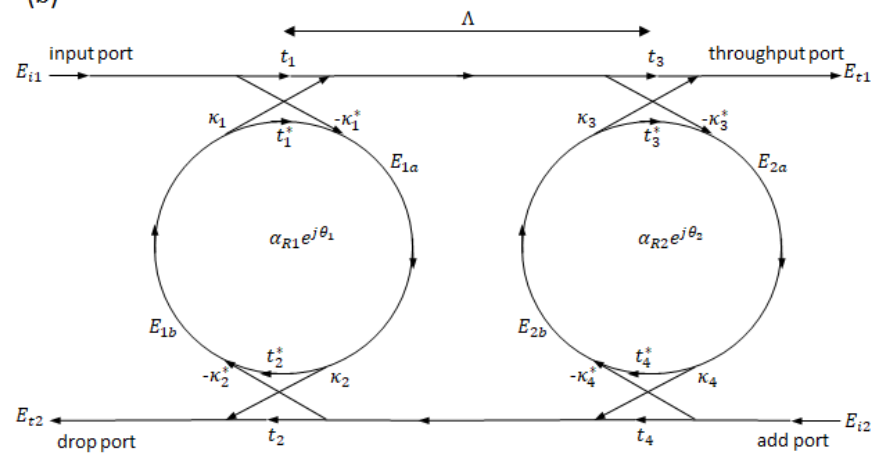

Figure 6. MRR configurations: (a) serially coupled configuration and (b) parallel coupled configuration.

obtained by choosing $\Lambda$ to be equal to an odd multiple of a quarter wavelength:

$$
\Lambda=(2 m+1) \frac{\lambda_{0}}{4 n_{W e f f}}
$$

Here, $\lambda_{0}$ is the center wavelength of the passband and $n_{W e f f}$ is the effective refractive index of the input waveguide [24]. If the length $\Lambda$ is chosen such that the $F S R$ of the ring resonators and the $F S R$ of the grating obey the following condition

$$
F S R=N_{\text {ring }} F S R_{\text {ring }}=M_{\text {grating }} F S R_{\text {grating }},
$$

the distance between the resonators can be calculated by

$$
\Lambda=\frac{M_{\text {grating }} n_{\text {eff }}}{N_{\text {ring }} n_{W e f f}} \pi r
$$

The parallel configuration has less restrictive requirement because it is not mandatory that each MRR resonances have to be precisely identical. In case of nonaligned resonant frequencies, multiple peaks, or ripple in the line-shape occur. 


\section{MATERIALS AND FABRICATION}

\section{A. General fabrication aspects}

Silicon technology was driver for electronic revolution of last decades and in particular CMOS technology. The use of these silicon process techniques for photonic applications requires dedicated SOI substrate for the integration of optical elements on a chip. The SPOI substrate consists of a single crystalline silicon layer on a buried oxide (BOX) and a thick silicon-handling substrate. This dedicated stack is considered as SOI wafer. The silicon-layer has typically a thickness of $220 \mathrm{~nm}$, the BOX of $2 \mu \mathrm{m}$ and in conjunction with the substrate the full wafer has a thickness of approximately $750 \mu \mathrm{m}$ for a wafer having a diameter of $200 \mathrm{~mm}$ and $1 \mathrm{~mm}$ for a wafer with a diameter of $300 \mathrm{~mm}$.

In the following, we describe exemplary a process flow for the realization of photonic structures in a silicon technology environment. A process flow consists of single process steps which are applied on planar surfaces as deposition of layers, e.g. by chemical- and physical vapour deposition (CVD/PVD), patterning by reactive-ion-etching (RIE) or wet-etching processes, chemical-mechanical-polishing (CMP) and others. One main condition is the realization of planar surfaces to transfer the pattern-information onto the wafer by means of optical lithography. Here the structural information of one layer of the chip, e.g. patterning of SOI-layer for waveguide patterning is placed on a photo mask. The information is transferred via optics into a photo-resist, which is chemical sensitive to the applied wavelength usually in the deep ultra-violet range of the light source. Due to the fact that the minimum resolution, also known as critical dimension (CD), is direct proportional to the applied wavelength, laser sources with a wavelength of $248 \mathrm{~nm}$ or $193 \mathrm{~nm}$ are typically used, e.g. a $K r F$-laser. Due to a fix depth of focus (DoF) from the optical system, the thickness of the applicable photo-resist is limited and it has to be coated homogeneously over the wafer to achieve reproducible and stable conditions. Hence, a surface with minimum topography is beneficial.

\section{B. Fabrication of silicon-based waveguides}

In the following, we consider a simple patterning of the silicon waveguide structure. The schematic process flow is shown in Figure 7. Optional a first dielectric protection layer can be deposited by CVD on the SOI-layer (not shown in Figure 7). A thermal oxidation of the silicon is usually avoided because of the inherent decrease of the silicon thickness and the related changes of the optical properties.This deposited layer supports to maintain the rectangle shape of the intended wave guide because it protects the top edges of the wave guide. Moreover the dielectric protection layer can be used as antireflective-coating to improve the lithography process, depending on the thickness and material. Subsequently the photoresist is deposited on the surface followed by an exposure and development process. Now the underlying layer can be patterned by a reactive-ion-etching process. RIE enables a strong anisotropic etching of the layers and allows steep and almost rectangular sidewalls. The RIE process can be tuned in a way that a high selectivity is achieved with respect to the BOX layer. A critical parameter for the optical performance is the roughness of the side-wall [29]. After patterning of the waveguide structures a simple passive photonic structure has been realized. Usually silicon wave guides are realized in a more complex technology platform together with structures for light coupling (e.g. grating couplers) or even more complex with active devices as photo detectors or modulators. For this reason the very simple presented wave guides have to be protected by a stack of different layers consisting usually of silicon oxides and silicon nitride. Figure 8 shows an example of an etched waveguide covered by a stack of $\mathrm{SiO} / \mathrm{SiN} / \mathrm{SiO} / \mathrm{SiN}$. However, depending on the overall integration scheme and additional devices, which have to be integrated afterwards, these photonic protection layers can appear in different stack compositions. A release process is required to use silicon waveguides for optical sensing. The complexity of this release process depends indeed on the stack composition of the protection layers. As an example, a waveguide that is covered by a silicon dioxide and a silicon nitride layer with a thickness of $10 \mathrm{~nm}$ and 100 $\mathrm{nm}$, respectively, is described in Ref. [30]. Alternatively, the silicon waveguide can be released from the backside of the wafer, which was recently demonstrated [31]-[34].

\section{Specific fabrication aspects for MRR}

As mentioned before, most critical for the reproducible realization of efficient MRRs on wafer-level is the gap between the straight waveguide and the ring. This reproducible and stable minimum distance is approximately $100 \mathrm{~nm}$ for process environments with a $248 \mathrm{~nm}$ DUV lithography. However, independently of the final exploited geometry, the key aspect in a MRR is how the coupling between the ring and the waveguide(s) is realized. There are two main configurations for this coupling [24]:

1) a vertical coupling configuration, where the bus waveguides are either on top or beneath the ring resonator;

2) a lateral coupling configuration, where the bus waveguides and the ring resonator are in the same plane.

The vertical coupling method allows the precise control of the coupling distance and of the coupling coefficient. To get this result, an accurate and reproducible fabrication technique is used in semiconductor-based RR such as the epitaxial growth. In this configuration it is possible to fabricate the ring and the bus waveguides out of separate materials which opens up the possibility of creating active ring or disk structures when using an appropriate material. Although vertically coupled MRR commonly require higher fabrication complexity because processes like wafer bonding, regrowth or re-deposition are required, they can allow for the realization of small radius MRRs.

The lateral coupling method has been widely exploited for realizing ring resonators with larger radius $(>100 \mu \mathrm{m})$. It uses a weak guiding waveguide layout where the waveguide mode confinement is still high enough to fabricate ring resonators with negligible bending loss. Ring resonators with small radii have also been fabricated utilizing the lateral coupling scheme by choosing a suitable waveguide design: less stringent fabrication requirements are requested for achieving a very small 


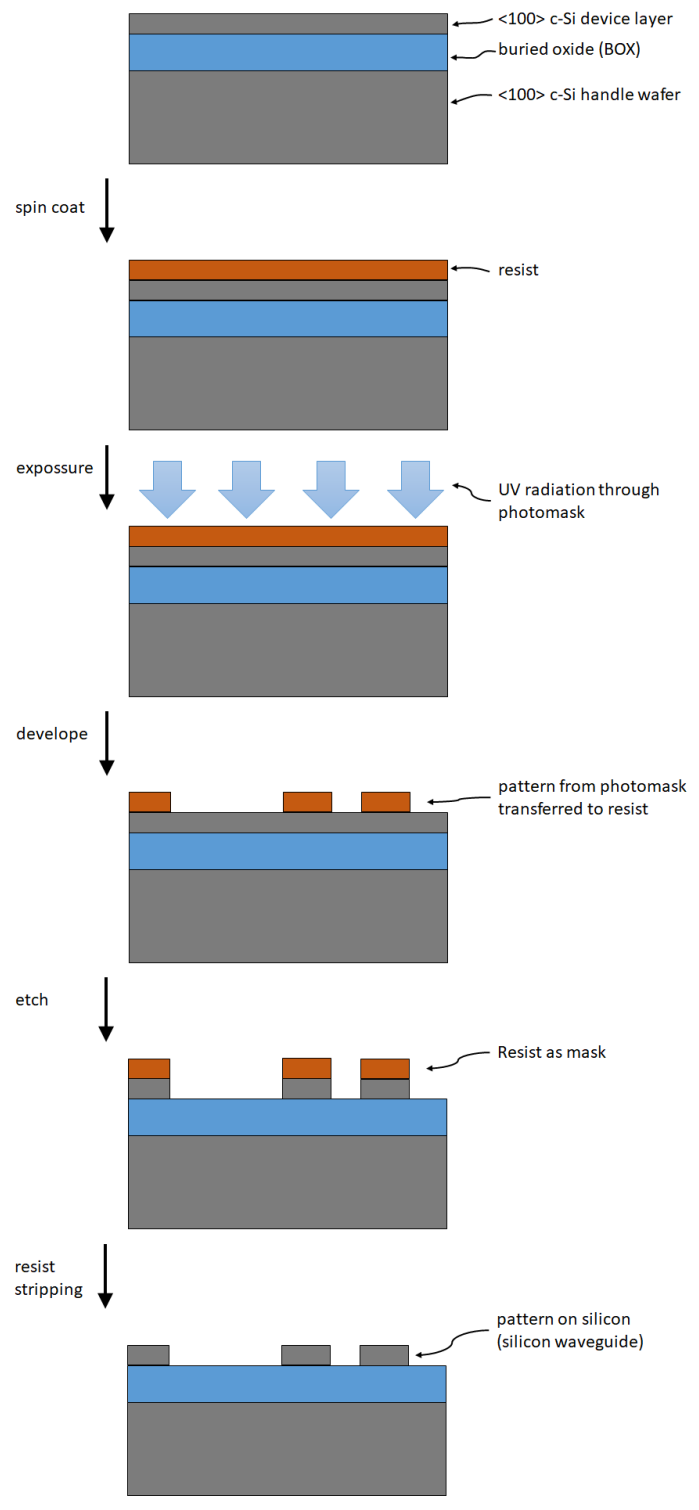

Figure 7. Schematic of a simplified fabrication flow.

coupling gap (usually much less than $1 \mu \mathrm{m}$ in the case of very strong guiding waveguides). The lateral coupling approach allows the use of multimode interference couplers with higher processing tolerances with respect to the power coupling coefficient which eliminates the fabrication of small $(<1 \mu \mathrm{m})$ and deep coupling gaps.

Both coupling schemes have advantages and disadvantages. Depending on the application, the MRR parameters must be adjusted to fit the desired requirements and, consequently, the choice of best fabrication technique must be adequately triggered achieving the best compromise. In case of siliconbased MRR, the fabrication process can be summarized as follows:

1) material preparation. Initially made of pure $\mathrm{Si}$, since the last decade the final system is commonly made of a multilayer structure where Si ring and waveguides are surrounded by low refractive index medium deposited/laying on $\mathrm{Si}$ substrate.

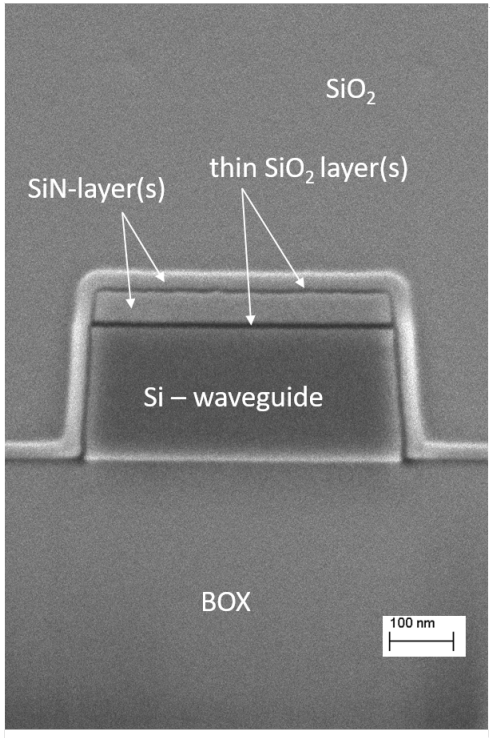

Figure 8. Scanning electron microscope cross-section of a patterned waveguide with different protection layers.

2) MRR layout definition by "drawing" on the material surface the MRR geometry pattern with a lithography process.

3) The realization of the RR structure by removing everything apart the RR pattern by the so called microstructuring techniques.

Figure 9 shows a fabricated photonic chip with MRR-based sensors. As far as the material preparation, the best MRR

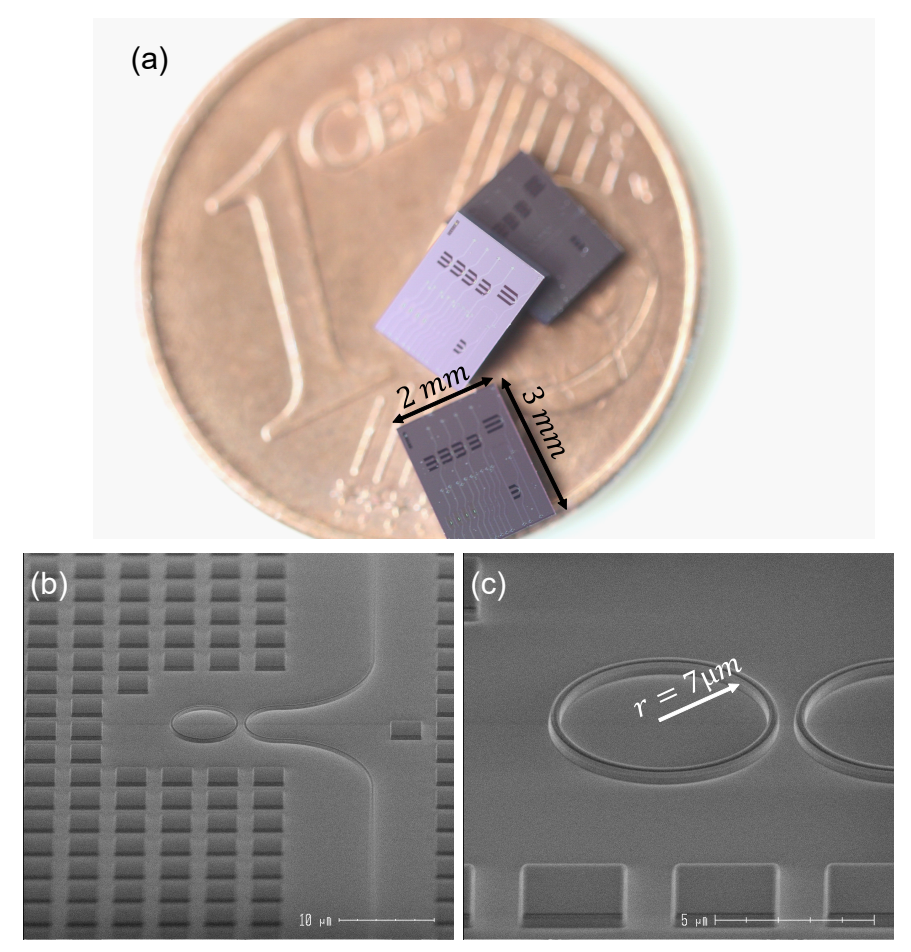

Figure 9. (a) Photonic Chip fabricated in a PIC technology. (b) Image of a silicon-based MRR recorded with a scanning electron microscope. (c) Magnification of the MRR with a radius of $7 \mu \mathrm{m}$. 
performances have been obtained on multilayer structures. Initially prepared by epitaxially growth by exploiting the hightech in $\mathrm{Si}$ microlectronics, in the last decade they have been achieved by the so-called wafer bonding technique. In this case a $\mathrm{Si}$ wafer is bonded to another semiconductor or insulator material and then patterned by suitable micro-structuring techniques. Depending on the use of an intermediate layer or not between the two bonded wafers, wafer bonding can be activated either by temperature or pressure or chemicals or plasma, respectively [24]. When an intermediate layer is not used, adhesion bonding or eutectic bonding are exploited. In case that an intermediate layer is applied, instead, the intermediate layer is deposited/realized on one wafer (normally the $S i$ one) and the fusion bonding and the anodic bonding are often used to achieve the bond between the two wafers. By combining suitable materials, wafer bonding allows for exploiting the advantages of using different materials while minimizing their disadvantages. Recently more sophisticated solutions have been proposed that combine the wafer bonding with ion implantation. The Smart-cut technology is one of these. In this case a $\mathrm{Si}$ wafer is oxidized at the surface to achieve a $\mathrm{SiO}_{2}$ surface layer (as alternatives, $\mathrm{SiO}_{2}$ layers can be deposited by Chemical Vapour Deposition [35]). The resulting $\mathrm{Si}$ (Substrate) $/ \mathrm{SiO}_{2}$ system undergoes to Ion Implantation (normally $\mathrm{H}$ or $\mathrm{He}$ ions that implant as a controlled depth from the surface) and then bonded to another Si wafer. After thermal treatment, in the ion implanted region, bubbles are formed and the multilayer can be cut there away due to the reduced mechanical resistance therein promoted. The splitting process leaves a $\mathrm{Si}$ (substrate) $/ \mathrm{SiO}_{2} / \mathrm{Si}$ multi-layer structure, commonly know as SOI that can then undergo to micro-structuring process to achieve the final MRR layout.

In order to get the final MRR layout on the wafer bonded multistack, micro-structuring advanced approaches have been finalized. Dry etching has been widely exploited to achieve the final MRR geometry by controlled material removal that leaves unaltered the MRR layout on the substrate. In this case the pattern of a ring resonator layout must be carefully transferred and in $S i$-technology this is achieved by the socalled right masking technique where unmasked regions are removed away. The ring and the coupled waveguide are therefore masked to prevent their etching whilst all the rest is removed (such as the cou-pling gap). There are three main categories of masking materials: metals $(\mathrm{Ni}, \mathrm{Cr}, \mathrm{NiCr}, \mathrm{Ti}$, $W, \mathrm{Pt})$, dielectrics $\left(\mathrm{SiO}_{x}, \mathrm{SiN}_{x}\right)$ and polymers (Photoresist, E-beam resist). Depending on the final application of the $S i$ based MRR, different mask types are used. In particular, metal masks are preferred when deep etching is needed because they are extremely etch resistant. However, they still present the drawback of being difficult to remove and the metal grains result in transferred striations. When nanostructures need to be included, dielectric masks are used because they provide good etch resistance and very little transferred striations to the underlying material. As alternatives, polymers have also been introduced as mask-materials thanks to their highly etching resistance when fully cured. Polymers normally are not used to etch high thicknesses $(>1 \mu \mathrm{m})$ and can suffer form the risk of contaminating the etch chamber. The lithography process has been also achieved by electron beam lithography (EBL). In this case, the pattern is defined by scanning an electron beam by a software-controlled procedure to design the MRR layout and a high spatial resolution can be obtained.

Once the MRR pattern transfer has been made, the etching process is applied: it can be isotropic and anisotropic. In the first case, the etching rate is the same in all directions. In the anisotropic case, instead, the etching rate is direction dependent. This is an important aspect in the fabrication of MRR, where deeply etched waveguides are needed to obtain a strong mode confinement of the field which in turn results in a small radius of curvature with acceptable loss. In order to perform the etching process, reactive ion etching (RIE) has been most widely used in $S i$-based MRR devices. Ions are accelerated towards the material to be etched, and the etching reaction is enhanced in the direction of travel of the ion. Different dry etching systems are available on the market apart conventional RIE, such as inductively coupled-plasma reactive ion etching (ICP-RIE), reactive ion beam etching (RIBE), or chemically assisted ion beam etching (CAIBE). Gases which are commonly used (combining several) in dry etching processes are methane $\left(\mathrm{CH}_{4}\right)$, hydrogen $\left(\mathrm{H}_{2}\right)$, hydrogen bromide $(\mathrm{HBr})$, nitrogen $\left(\mathrm{N}_{2}\right)$, chlorine $\left(\mathrm{Cl}_{2}\right)$, silicon tetrachloride $\left(\mathrm{SiCl}_{4}\right)$, $\operatorname{argon}(\mathrm{Ar})$, and xenon $(\mathrm{Xe})$.

One of the first and smallest MRRs in the $\mathrm{Si}_{-} \mathrm{SiO}_{2}$ material system which is also known as SOI is dated back to 1998 thanks to the pioneering work of Little and coworkers [36]. Made of a ring with radii of 3,4 , and $>5 \mu \mathrm{m}$, the devices showed quality factors up to 250, and an FSR of 20, 24, and $>30 \mathrm{~nm}$ for a wavelength of $>1550 \mathrm{~nm}$, respectively. The intensity difference between the on-resonance and the off-resonance state is measured to be more than $15 \mathrm{~dB}$. The fabrication process is normally made up of a deposition of around $>1 \mu \mathrm{m}$ thick buffer layer of $\mathrm{SiO}_{2}$, followed by a deposition of a waveguiding core layer of amorphous $\mathrm{Si}$ with a typical thickness of $0.2 \mathrm{~m}$. The amorphous $\mathrm{Si}$ is annealed to convert it to poly-crystalline silicon out of which the ring and bus waveguides are made (typically values are: width close to $>0.5 \mu \mathrm{m}$, height close to $>0.2 \mu \mathrm{m}$ ) then patterned using a plasma etch process (the most diffused is an $\mathrm{HBrCl}$ ). The index contrast can be quite high (even $3.5: 1.5$ or $3.5: 1.0$ depending on whether the mate-rial external to the $S i$ waveguide is $\mathrm{SiO}_{2}$ or air, respectively). The width of the waveguide should be less than $>600 \mathrm{~nm}$ in order to achieve single mode operation, commonly $>450 \mathrm{~nm}$ are quite well used. Other technologies of fabricating MRRs are: UV lithography with an illumination wavelength of $>248 \mathrm{~nm}$ has been used to achieve MRRs with similar dimensions and figures. Several improvements on the processes have been proposed in the last years in order to optimize the ring performances even for decreasing the ring size [37]-[39].

\section{OPTIMIZATION OF MICRO-RING RESONATORS FOR SENSING}

Integrated photonic sensors are optical transducers that convert the presence of an analyte into a property of light, i.e. intensity, phase, or wavelength. From practical point of view, 
it is useful to distinguish between the waveguide sensitivity and the ring resonator sensitivity because the overall sensor performance can be optimized by taking both into account.

In this section, we introduce waveguide sensitivity, ring resonator sensitivity and the overall sensitivity and we provide a guideline to improve each.

\section{A. Waveguide sensitivity}

In contrast to more traditional free-space optical sensors, integrated photonic sensors based on MRR use light that is confined inside an optical waveguide. As opposed to optical fibers, an integrated optical waveguide has a rectangular shape due to the fabrication, as explained in section III. In general, three waveguide structures have been intensively studied over the last two decades. The most common type is known as strip waveguide. It has a rectangular form with a height of $220 \mathrm{~nm}$ and a width ranging from $450 \mathrm{~nm}$ to $500 \mathrm{~nm}$.

The main contribution to the optical losses comes from sidewall roughness. As shown in Figure 10, the lowest loss can be achieved with rib waveguides, while the loss increases with waveguide structures having larger sidewall areas. For example, for a strip waveguide with a cross-section of $500 \mathrm{~nm}$ times $220 \mathrm{~nm}$, light scattering at the sidewalls leads to an optical loss of about $2 \mathrm{~dB} / \mathrm{cm}$ [40]. As rule of thumb the optical losses scale with the optical field confinement, i.e. the more light is confined within the waveguide, the lower are the optical losses. The optical confinement is the fraction of light inside the waveguide-core [41]. It is convenient to calculate the cross-sectional power to represent the light inside a specific area, e.g. in a waveguide core. It can be estimated by considering the Poynting vector, which represents the instantaneous power flow due to instantaneous electric and magnetic fields. However, if we consider an electromagnetic waves that is represented as sinusoidally varying fields at a specified frequency, the time-averaged Poynting vector is used. The cross-sectional power is then expressed as the time-averaged complex Poynting vector integrated over the waveguide cross-section

$$
P=\iint \frac{1}{2} \operatorname{Re}\left\{\mathbf{E} \times \mathbf{H}^{*}\right\} \mathrm{d} x \mathrm{~d} y,
$$

where $E$ and $H$ are the electric and magnetic field vectors, respectively, and $e_{z}$ is the unit vector in $z$ direction. Finally, the field confinement factor, also known as field interaction factor, is estimated as

$$
\Gamma=\frac{P_{\text {core }}}{P_{\infty}},
$$

where $P_{\text {core }}$ is the cross-sectional power inside the core, i.e. the optical power confined inside the core, and $P_{\infty}$ represents the complete power, that means the power over the entire cross-section [42].

The interaction of the guided light with the surrounding medium is not only described by the field confinement factor but also by the waveguide sensitivity. It can be shown that a higher field confinement inside the cladding material, which is in fact the fluid, increases the waveguide sensitivity [43], [44]. Since the effective refractive index reflects the light

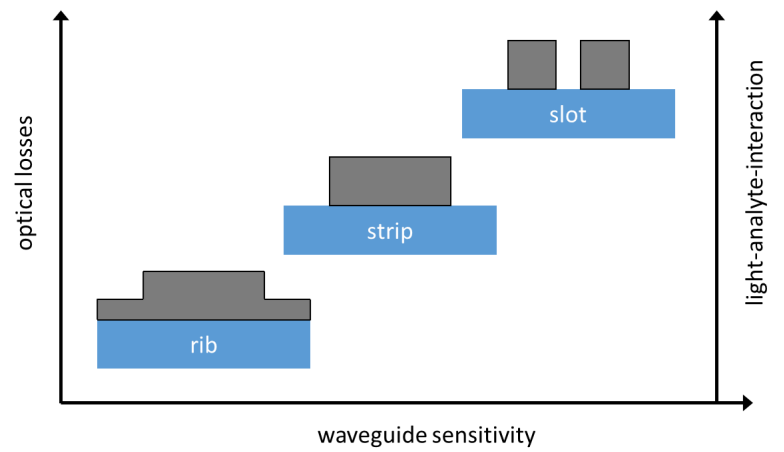

Figure 10. Typical waveguide geometries are rib, strip and slot waveguides. The increased light-analyte-interaction increases on the one hand the waveguide sensitivity but increases, on the other hand, the optical losses, too.

propagation inside a waveguide, the waveguide sensitivity is defined in different ways, depending on the sensing mechanism, i.e. chemical bulk sensing or label-free surface sensing. In principle, the waveguide sensitivity is evaluated by

$$
S_{w g}=\frac{\Delta n_{e f f}}{\Delta \xi}
$$

where $\Delta n_{\text {eff }}$ represents the effective refractive index change and $\xi$ represents either the change of the cladding refractive index $n_{\text {clad }}$ in case of chemical bulk sensing or the thickness of the adhered layer $t$ or the mass density $\rho$ when label-free surface sensing is considered. Due to the different units of the considered sensing mechanism $\xi=\left\{n_{\text {clad }}, t, \rho\right\}$, the unit of the waveguide sensitivity is given by $R I U / R I U, R I U / \mathrm{nm}$ or $R I U /\left(\mathrm{pg} / \mathrm{mm}^{2}\right)$, respectively, where $R I U$ is the refractive index unit.

It is common to optimize the waveguide sensitivity by calculating the optical field distribution with, e.g., by using the finite-element method. In this case, the effective refractive index $n_{\text {eff }}$ can be calculated by solving the Helmholtz equation for different $n_{\text {clad }}$. By plotting $n_{\text {eff }}$ over $n_{\text {clad }}$ gives us $S_{w g}$ by taking the slope of this graph [31].

Preferred waveguide structures to enhance $S_{w g}$ are slot waveguides or subwavelength gratings (SWG) [45], [46]. Besides that, the use of the transverse-magnetic (TM) instead of transverse-electric (TE) mode can also increase the waveguide sensitivity [47]. The polarization of the guided waveguide mode influences the light-analyte-interaction. In general, both fundamental modes can be used, i.e. the first TE mode and first TM mode. In case of the fundamental TE mode, the dominant transverse electric field component is oriented parallel to the substrate, while in case of the fundamental TM mode the dominant transverse magnetic field component lies parallel to the substrate. The TM mode shows higher light-analyteinteraction at the expense of optical losses. Besides that, the TE mode is preferred because most photonic devices like beam splitter, grating coupler or spot-size converter are optimized for it since it is commonly used for applications in telecommunication, a technological driver of PICs. 


\section{B. Ring resonator sensitivity}

The resonance wavelength shift can be calculated from resonator metrics, that is [48]

$$
\Delta \lambda_{\text {res }}=\frac{\lambda_{\text {res }}}{n_{g}} \Delta n_{\text {clad }},
$$

where $n_{g}$ is the group index, $\lambda_{\text {res }}$ is the resonance wavelength and $\Delta n_{\text {clad }}$ determines the refractive index change of the cladding material. The group index can be calculated by

$$
n_{g}=n_{e f f}-\lambda \frac{\delta n_{e f f}}{\delta \lambda} .
$$

Here, the effective refractive index of the optical waveguide is denoted as $n_{\text {eff }}$. The second term of Eq. 41 is referred to as dispersion of the group index. Assuming a small resonance wavelength shift and, hence, a flat dispersion, the group index can be approximated by

$$
n_{g} \approx n_{\text {eff }}=\frac{\lambda^{2}}{F S R} \frac{1}{L_{\text {ring }}},
$$

where $L_{\text {ring }}$ is the ring circumference and $F S R$ is the free spectral range. From this formula it is apparent that the free spectral range has a quadratic dependency on the wavelength, i.e. $F S R \propto \lambda^{2}$.

However, the ring resonator sensitivity depends not only on the waveguide geometry and, therefore, a second definition defining the ring resonator sensitivity is given by

$$
S_{r r}=\frac{\Delta \lambda_{r e s}}{\Delta n_{e f f}} .
$$

\section{Overall sensor performance}

Taken both definitions into account, we get the overall photonic device sensitivity defined by

$$
S=S_{w g} S_{r r}=\frac{\Delta n_{e f f}}{\Delta \xi} \frac{\Delta \lambda_{\text {res }}}{\Delta n_{\text {eff }}}=\frac{\Delta \lambda_{\text {res }}}{\Delta \xi} .
$$

It should be noted that the change of the cladding refractive index $\Delta n_{\text {clad }}$ is induced by binding of antigens to the functionalized waveguide surface. These definitions, however, are solely related to the photonic device and not to a directly measurable quantity. In this scenario, the minimum detectable change in the cladding refractive index gives us the limit of detection (LOD), which depends clearly on the minimum detectable resonance wavelength shift $\Delta \lambda_{\min }$ that can be resolved by the measurement set-up.

The $L O D$ can be determined by

$$
L O D=\Delta \xi .
$$

Therefore, the $L O D$ can have three different units, depending on $\xi$. For chemical bulk sensing, a minimum change of the refractive index in the cladding ( $\left.\xi=\Delta n_{\text {clad }}\right)$ is considered and the unit is RIU. In case of label-free surface sensing, the minimum change of the thickness of the adhere layer $(\xi=\Delta t)$ or of the mass density $(\xi=\Delta \rho)$ is considered and the unit of the $L O D$ becomes $\mathrm{pg} / \mathrm{mm}^{2}$. The $L O D$ can also be calculated from the minimum resonance wavelength shift $\Delta \lambda_{\min }$ and the sensitivity $S$ by

$$
L O D=\frac{\Delta \lambda_{\min }}{S} .
$$

To get a metric which is independently from the measurement set-up, an intrinsic $L O D$ ( $i L O D)$ is necessary [49]. It can be obtained by setting the measurement resolution $M R$ as full width at half maximum $(F W H M)$ of the resonance peak, which leads to

$$
i L O D=\frac{F W H M}{S}=\frac{\lambda_{r e s}}{Q S},
$$

where $Q$ denotes the optical quality factor, which is determined by

$$
Q=\frac{\lambda_{\text {res }}}{F W H M} .
$$

In the following, we provide information that should be considered when designing photonic sensors based on MRR:

1) Increasing the waveguide sensitivity $S_{w g}$ increases the light-analyte-interaction. In fact, this determines the wavelength shift $\Delta \lambda$ and has a strong impact on the overall sensitivity. This can be achieved by a simple simulation study, in which the waveguide geometry is chosen to obtain highest $S_{w g}$.

2) The use of the transverse-magnetic (TM) instead of transverse-electric (TE) mode can also increase the waveguide sensitivity due to a higher field confinement inside the aqueous solution.

3) An enhanced waveguide sensitivity only improves the $L O D$ if it does not result in additional losses of the waveguide mode [50].

4) Enhancing the ring resonator sensitivity $S_{r r}$, which determines the wavelength shift $\Delta \lambda$ depending on the effective refractive index change $\Delta n_{e f f}$, which can be also done by simulating the spectrum of the MRR at different $\Delta n_{\text {eff }}$.

5) The impact of noise on the determination of the resonance wavelength will be reduced with increased optical Qfactor [51], [52]. Therefore, it is necessary to reduce optical losses in order to decrease $F W H M$. A lower $F W H M$ is lowering the attenuation inside the MRR and, therefore, minimizes the smallest detectable wavelength shift $\Delta \lambda$ and consequently the $M R, L O D$ as well as $i L O D$.

6) Reducing the small footprint by decreasing the circumference $L_{\text {ring }}$ reduces the area consumption and, as a direct consequence, device costs significantly. This leads to a high integration density, which is of special interest for multiplexing.

7) Small ring radii increase the free spectral range FSR. This is from great interest if large molecules are detected, leading to a large resonance shift $\Delta \lambda_{\text {res }}$. The $F S R$ needs to be larger than $\Delta \lambda_{\text {res }}$, which is achieved by reducing the ring circumference.

8) On the other hand, a small ring circumference reduces the ring resonator sensitivity and limit of detection. Therefore, a trade-off between sensitivity and free spectral range is required, which strongly depends on the application.

9) The $i L O D$ improves by reducing the losses, which is function of the light wavelength. Therefore, photonic 

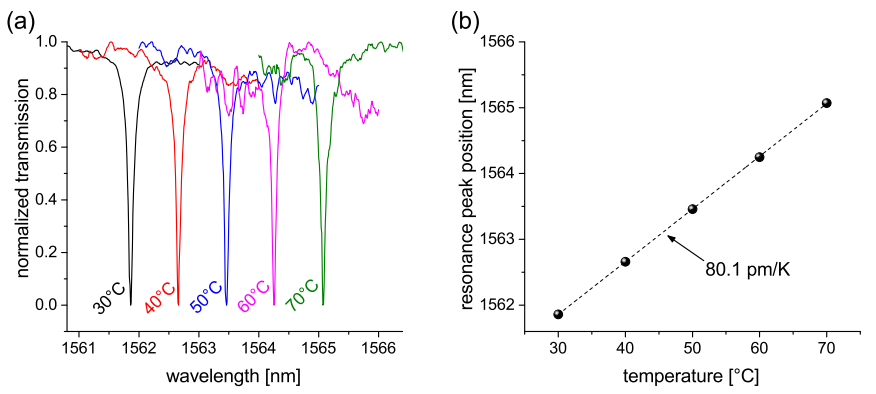

Figure 11. a) Normalized transmission of a MRR at various temperature. b) Resonance peak position as function of the temperature.

biosensors should operate in the visible, where the water absorption is much lower than in the infrared [50].

As mentioned before, each waveguide-type has advantages and disadvantages and therefore, a design trade-off regarding sensitivity and optical losses is necessary. Recently, a hybridwaveguide ring resonator was proposed to combine a strip waveguide with a slot waveguide in such a way that the figure of merit $F O M=S_{r r} / F W H M$ is maximized [53].

Another important sensitivity definition is required due to the strong thermo-optical effect of silicon. Please note, it is common to neglect the thermal expansion of silicon $\left(\kappa_{S i}=3.57 \cdot 10^{-6} / K\right)$ since it is about 100 times smaller than the thermo-optical effect $\left(d n / d T=1.8 \cdot 10^{-4} / K\right)$ [54]. The thermo-optical effect changes the effective refractive index $n_{\text {eff }}$ and, hence, the a resonance wavelength shift $\Delta \lambda_{\text {res }}$ occurs. As rule of thumb, the more light is confined inside the silicon waveguide, the larger is $\Delta \lambda_{\text {res }}$. In other words, a rib waveguide shows a stronger dependence of the effective refractive index on the temperature as a slot waveguide because the guided light has a higher interaction with the silicon. The resonance wavelength shift $\Delta \lambda_{\text {res }}$ has a strong linear dependency on the temperature $T$. The temperature-sensitivity is therefore defined as follows:

$$
S_{T}=\frac{\Delta \lambda_{r e s}}{\Delta T}
$$

Figure $11 \mathrm{~b}$ shows an example. The data of Figure $11 \mathrm{~b}$ is deduced from Figure 11a. A temperature-sensitivity of $80.1 \mathrm{pm} / \mathrm{K}$ is revealed from the slope in Figure $11 \mathrm{~b}$.

\section{Surface Functionalization FOR LABEL-FrEE DETECTION}

Label-free assays are preferably used because they allow for direct measurement of the target eliminating labelling steps and therefore reduce effort for sample preparation. In this way, even targets are measured which cannot be labelled easily and analytes can be detected in the native state [55], [56].

If we consider a basic label-free assay, a sensor surface is coated with an affinity structure, which binds the target, e.g. a virus, at low concentrations with high selectivity (see Figure 12). The resulting mass gain or local change of the refractive index is detected by the MRR, and a signal shift is obtained. Therefore, two key steps must be met: a) modification of the sensor surface to allow for controlled surface chemistry and b) immobilization of a suitable high-affinity binder on the surface while maintaining its activity.

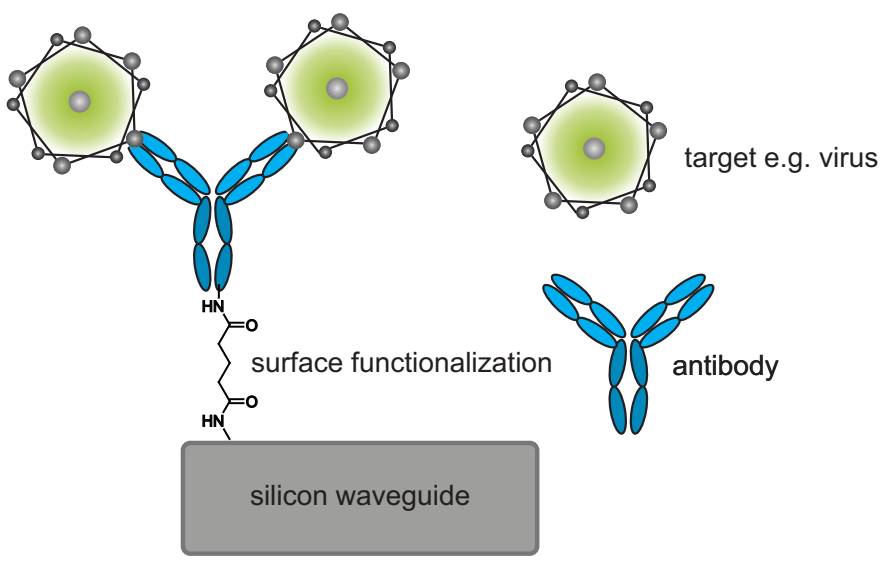

Figure 12. Basic schematic view of a label-free assay with antibodies immobilized on a silicon structure by a linker. The refractive index around the silicon structure is increased when the target is bound (not to scale).

\section{A. Surface modification}

Surface functionalization of silicon can be achieved with established organofunctional silane [55], [57], [58], which is based on the formation of covalent $\mathrm{Si}-\mathrm{O}-\mathrm{Si}$ bonds between oxidized silicon structure and the coating substance. Silicon has a thin native oxide layer, if exposed to air and/or moisture [59]. Therefore, a condensation type-reaction of oxidized bulk silicon surface and pre-hydrolyzed alkoxysilanes is typically used. In the first step, the surface of the silicon may be cleaned and activated, e.g. by strong heating [58], with plasma cleaning [60] or chemically with strongly acidic hydrogen peroxide solution (Piranha solution) to yield a pristine surface with silanol groups [61]-[63], as shown in Figure 13a. In the second step, either methoxysilanes (Figure 13b) or ethoxysilanes in a pure solvent or with a small percentage of water [64] (to in situ forms the reactive silanes) are added. Common methoxy silanes are (3-aminopropyl)-trimethoxysilane (APTMS), which leads to amino-modified surfaces [55] or (3-glycidyloxypropyl)-trimethoxysilane (GLYMO) to obtain epoxy-modified surfaces [62] (see Figure 13c). After an incu-

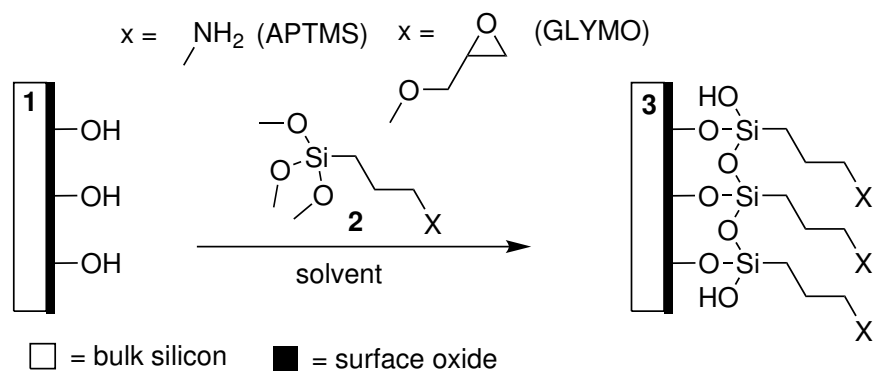

Figure 13. Reaction of the oxidized silicon surface (1) with methoxysilanes (2) to obtain a functionalized surface (3).

bation ranging from minutes to hours, the sample is thoroughly washed and dried; an additional curing step at increased temperature may be performed to aid the condensation reaction 
[58], [64]. The obtained functionalized surface is now available for further modifications (Figure 13c).

\section{B. Immobilization of high-affinity binder}

Several binder types like peptides [65], aptamers [64] or molecular imprinted polymers (MIPs) [66] are used for sensor surfaces coatings, but the most prominent class are antibodies [63] or antibody fragments [58]. Antibodies are Y-shaped proteins (see Figure 12) and very sensitive and selective to the target structure rendering them the ideal choice for many biosensor applications. Typically, these proteins are covalently immobilized by coupling accessible amino groups from lysine residues or if present, free sulfhydryl groups from cysteine residues on reactive surfaces. For amine surfaces, various coupling paths have been demonstrated using an amine group of the protein and a linker molecule. The most prominent are hereby introduced (Figure 14):

1) Glutaraldehyde (GAD) activation of the surface followed by coupling with the target protein $(\mathrm{P})$ and final reduction of the generated imine-bonds to yield stable amine-bonds with sodium cyanoborohydride $(\mathrm{NaBH} 3(\mathrm{CN}))$ [58].

2) Succinic anhydride (SCA) modification of the surface and activation of the carboxylic acid with NHS/EDC (NHS $=$ N-Hydroxysuccinimid, EDC = 1-Ethyl-3-(3dimethylaminopropyl)carbodiimid) to yield an NHS ester subsequently reacting with free amines of the protein to yield stable amide bonds [55].

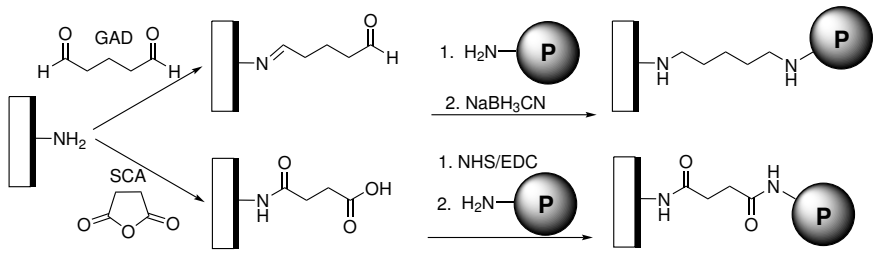

Figure 14. The aminated surface is either reacted with glutaraldehyde (top), or with or succinic anhydride (bottom) and then coupled to the protein.

While the results of both reaction paths seem very similar, surface charges can play an important role in the correct orientation of the immobilized proteins [67]. An alternating head-on tail-on immobilization pattern could be observed for antibodies in the SCA path, due to the neutral surface charge of NHS activated surfaces at neutral $\mathrm{pH}$, or in the conjugation step [67]. In the GAD path, the final surface may be positively charged due to the generated secondary amines, possibly leading to increased unspecific binding of proteins in the final assay. Also, higher head-on immobilization for antibodies was observed for GAD surfaces, possibly due to the more reactive $\alpha$-amino groups in the head [67]. The direct immobilization of the antibody may lead to deformation and loss of function of the antibody. Therefore, direct immobilization of the antibody should be balanced against the immobilization with more robust affinity proteins like Protein A or Protein G [69]. Oriented immobilization of antibodies on the surface might be favorable if the highest activity of the surface is desired [67], [70]. With new protocols, as illustrated in Figure 15, the benefits of antibodies orientation with affinity a)

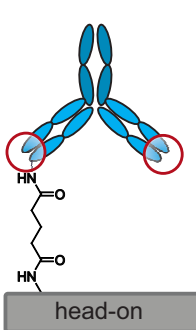

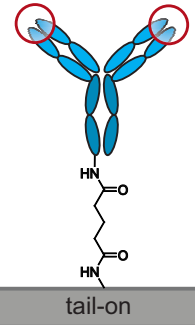

Figure 15. Head-on (a), tail-on (b) and oriented (c) immobilization with the affinity binder Protein A of IgG [68]. The antibody binding sites are highlighted in red.

proteins and covalent immobilization were shown as promising immobilization strategies for future biosensors [68].

\section{Recipe for label-free surface sensing}

In the following we want to provide step-by-step instructions for a specific surface modification of a micro-ring resonators chip with sensitivity to trinitrophenyl. This recipe can be used as guideline and starting point for antibody-based biosensors. The coating with Protein-G allows for convenient affinity binder immobilisation on the sensor surface. While we used anti TNT-antibodies, due to the high affinity of Protein-G to various IgG species and classes the chip can be readily modified with other high affinity antibodies to match the required target molecule. Also, antibody immobilisation on the Protein-G layer may be directly monitored to optimise experimental setups.

1) Cleaning and activation (optional):

The photonic chip should carefully vortexed for at least $20 \mathrm{~s}$ with $1 \mathrm{ml}$ of $1 \mathrm{M} \mathrm{NaOH}$ in a $2 \mathrm{ml}$ tube at $1000 \mathrm{rpm}$ and incubated for five minutes. Subsequently, the chip should be carefully vortexed in water for $20 \mathrm{~s}$ and incubated for five minutes. The chip is transferred in $1 \mathrm{M} \mathrm{HCl}$, vortexed carefully for $20 \mathrm{~s}$, and incubated for another five minutes. Finally, the chip can be incubated in water for five minutes, vortexed in absolute ethanol, followed by acetone (HPLC grade), and then allowed to dry on a clean bench.

2) Silanization:

$10 \mu \mathrm{l}$ of (3-glycidoxypropyl)methyl dimethoxy-silane and $10 \mu \mathrm{l}$ water should be added to $980 \mu \mathrm{l}$ absolute ethanol and then mixed. The dry chip was submerged in the solution, carefully vortexed, and incubated overnight. Subsequently, the chip should be washed three times with approximately $1 \mathrm{ml}$ absolute ethanol and allowed to dry on a clean bench.

3) Affinity coating with Cys-Protein-G:

A $0.5 \mathrm{mg} / \mathrm{ml}$ solution of Cys-Protein-G in PBS $(100 \mathrm{mM}$ phosphate, $\mathrm{pH}=7.8$ ) needs to be prepared, and $100 \mu \mathrm{l}$ should be deposited on the chip.

4) Antibody immobilization:

The antibody of choice is the monoclonal mouse antibody A1.1.1 (subclass IgG1), which should be diluted to $1 \mathrm{mg} / \mathrm{ml}$ in PBS. About $100 \mu \mathrm{l}$ can be deposited on 
the chip as described above and incubated for one hour. Alternatively, human, goat, mouse or rabbit antibodies are suitable and allow for a broad variety of targets.

\section{EXPERIMENTAL SET-UPS AND MEASUREMENT PROCEDURE}

The light is introduced into the chip by means of grating coupler technique, i.e. the light is coupled from a single-mode fiber into a single-mode silicon waveguide via optical grating coupler, which are etched into the waveguide [71]. In the case of butt coupling, the light is introduced on the edge of the chip [72]. Butt coupling is typically utilized for applications in telecommunication since it provides low coupling-loss, large spectral bandwidth, and low-sensitivity to polarization [73]. For biosensing applications, it allows to couple TE and TM modes to the waveguide. TE modes are preferred when grating couplers are used due to the lower insertion loss. However, the main advantage of grating couplers is the possibility of waferlevel testing [74]. Due to this reason, the TE mode is typically used in PIC-technology, including biosensing applications. A comprehensive overview of coupling strategies can be found in Ref. [75].

For the application of MRR as chemical and/or biochemical sensor, three MRR are necessary. The first MRR is encapsulated in such a way that it can be used to track $\Delta \lambda$ due to a change of the temperature. This is necessary because silicon exhibits inherently a large thermo-optical effect, leading to change of the resonance condition [76], which can be advantageously used as temperature sensor [77]. However, by tracking the resonance shift with an encapsulated MRR, as shown in Figure 11a, allows to eliminate this dependency from the actual MRR-sensor, while both MRR should be placed near to each other. Such initial tests can be performed by using a hot plate if no heater is integrated into the photonic chip. The second MRR is not encapsulated and acts as the aforementioned sensor, which consists of the silicon-waveguide with fully active surface functionalization, as described in section V. The third MRR exhibits no surface functionalization and aims to measure the bulk refractive index.

Label-free detection requires a differentiation between the target analyte and the sample solution (background). Therefore, a rinsing step is typically used to obtain the resonance wavelength shift induced by the target analyte, as indicated in Figure 16.

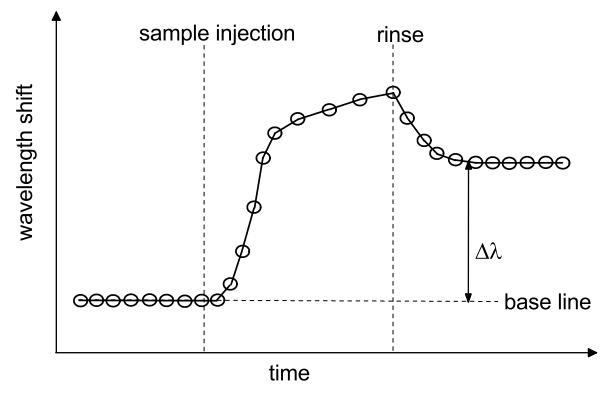

Figure 16. Real-time measurement of analyte binding.
Bulk sensing is typically performed by using, for example, different concentration of $\mathrm{NaCl}$ in DI water, as shown in Figure 17. From such measurement, the resonance wavelength shift can be deduced and plotted as function of the different concentrations. To obtain the ring resonator sensitivity $S_{r r}$, the concentration needs to be translated into a refractive index. The refractive index $n_{f}$ of the $\mathrm{NaCl}$ solved in DI water at different concentrations $C(w t \%)$ and at a wavelength of $1550 \mathrm{~nm}$ can be calculated by [78]-[80]

$$
n_{f}(w t \%)=1.3105+0.17151 \frac{C(w t \%)}{100} .
$$

This gives us a linear relation between the resonance wavelength shift to the refractive index of the aqueous solution, as shown in Figure 18. The slop of the graph in 18 gives us the ring resonator sensitivity $S_{r r}$.

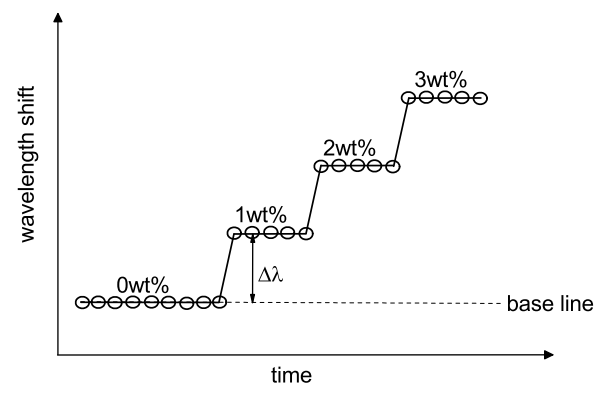

Figure 17. Measurement of the resonance wavelength shift due to different concentration of $\mathrm{NaCl}$ dispersed in water.

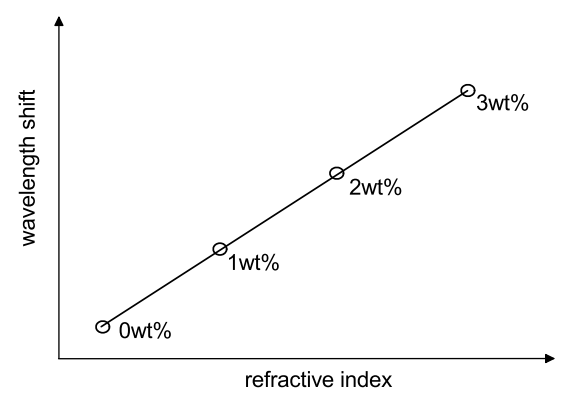

Figure 18. Mean value of the resonance wavelength shift, that can be deduced from Figure 17, over the refractive index of the sample solution.

\section{CONCLUSION}

In this tutorial we have introduced the fundamental theory of MRRs and their application as biochemical sensors. An overview of fabrication aspects is provided. Besides that, we have discussed the definitions of waveguide sensitivity and ring resonator sensitivity. We inferred that the increased waveguide sensitivity increases the light-analyte-interaction but it only improves the limit of detection if it does not result in additional optical losses. Further, we can conclude that a trade-off between ring resonator sensitivity and free spectral range is required, which strongly depends on the application. It was highlighted that the choice of waveguide geometry is significant for the ring resonator sensitivity. A guideline to 
design MRRs for biochemical sensing is provided and typical experimental procedures are discussed. MRR-based photonic sensors give perspective for future on-site diagnostics. However, future developments should tackle issues in terms of chip packaging. The practical use is further hindered by expensive laser sources and the large thermo-optical effect in silicon. Another bottle-neck is the fiber-to-chip light coupling, which requires a precise alignment of the optical fiber to the grating coupler. A pluggable solution is required for practical applications, in particular for disposable sensors. Those drawbacks need to be overcome and are topic of ongoing research. Overall, the tutorial provided a starting point for scientists in this interdisciplinary field and gives a critical overview ranging from fabrication methodologies and sensor optimization to surface functionalization and measurement procedures.

\section{REFERENCES}

[1] M. Angelopoulou, P. S. Petrou, I. Raptis, K. Misiakos, E. Livaniou, E. Makarona, and S. Kakabakos, "Rapid detection of mozzarella and feta cheese adulteration with cow milk through a silicon photonic immunosensor," Analyst, vol. 146, pp. 529-537, 2021. [Online]. Available: http://dx.doi.org/10.1039/D0AN01706J

[2] R. Guider, D. Gandolfi, T. Chalyan, L. Pasquardini, A. Samusenko, G. Pucker, C. Pederzolli, and L. Pavesi, "Design and optimization of sion ring resonator-based biosensors for aflatoxin $\mathrm{m} 1$ detection," Sensors, vol. 15, no. 7, pp. 17300-17312, 2015.

[3] M. S. McClellan, L. L. Domier, and R. C. Bailey, "Label-free virus detection using silicon photonic microring resonators," Biosensors and Bioelectronics, vol. 31, no. 1, pp. 388-392, 2012.

[4] M. S. Luchansky and R. C. Bailey, "Silicon photonic microring resonators for quantitative cytokine detection and t-cell secretion analysis," Analytical chemistry, vol. 82, no. 5, pp. 1975-1981, 2010.

[5] _ - "Rapid, multiparameter profiling of cellular secretion using silicon photonic microring resonator arrays," Journal of the American Chemical Society, vol. 133, no. 50, pp. 20 500-20 506, 2011, pMID: 22040005. [Online]. Available: https://doi.org/10.1021/ja2087618

[6] B. Chocarro-Ruiz, A. Fernández-Gavela, S. Herranz, and L. M. Lechuga, "Nanophotonic label-free biosensors for environmental monitoring," Current opinion in biotechnology, vol. 45, pp. 175-183, 2017.

[7] R. Chandrasekar, Z. J. Lapin, A. Nichols, R. Braun, and A. W. Fountain, "Photonic integrated circuits for department of defense-relevant chemical and biological sensing applications: state-of-the-art and future outlooks,' Optical Engineering, vol. 58, no. 2, p. 020901, 2019.

[8] C. Mai, P. Steglich, M. Fraschke, and A. Mai, "Back-side release of slot waveguides for the integration of functional materials in a silicon photonic technology with a full beol," IEEE Transactions on Components, Packaging and Manufacturing Technology, vol. 10, no. 9, pp. 1569-1574, 2020.

[9] R. Moretta, L. De Stefano, M. Terracciano, and I. Rea, "Porous silicon optical devices: recent advances in biosensing applications," Sensors, vol. 21 , no. 4 , p. $1336,2021$.

[10] S. Arshavsky-Graham, N. Massad-Ivanir, E. Segal, and S. Weiss, "Porous silicon-based photonic biosensors: Current status and emerging applications," Analytical chemistry, vol. 91, no. 1, pp. 441-467, 2018.

[11] M. A. Porcel, A. Hinojosa, H. Jans, A. Stassen, J. Goyvaerts, D. Geuzebroek, M. Geiselmann, C. Dominguez, and I. Artundo, "Silicon nitride photonic integration for visible light applications," Optics \& Laser Technology, vol. 112, pp. 299-306, 2019.

[12] P. Steglich, M. Hülsemann, B. Dietzel, and A. Mai, "Optical biosensors based on silicon-on-insulator ring resonators: A review," Molecules, vol. 24, no. 3, p. 519, 2019.

[13] A. Rahim, J. Goyvaerts, B. Szelag, J.-M. Fedeli, P. Absil, T. Aalto, M. Harjanne, C. Littlejohns, G. Reed, G. Winzer, S. Lischke, L. Zimmermann, D. Knoll, D. Geuzebroek, A. Leinse, M. Geiselmann, M. Zervas, H. Jans, A. Stassen, C. Domnguez, P. Muoz, D. Domenech, A. L. Giesecke, M. C. Lemme, and R. Baets, "Open-access silicon photonics platforms in europe," IEEE Journal of Selected Topics in Quantum Electronics, vol. 25, no. 5, pp. 1-18, 2019.
[14] A. Mai, P. Steglich, C. Mai, S. Simon, and R. Scholz, "Electronicphotonic wafer-level technologies for fast prototyping and application specific solutions," in 2019 PhotonIcs \& Electromagnetics Research Symposium-Spring (PIERS-Spring). IEEE, 2019, pp. 249-255.

[15] E. Luan, H. Shoman, D. M. Ratner, K. C. Cheung, and L. Chrostowski, "Silicon photonic biosensors using label-free detection," Sensors, vol. 18, no. 10, p. 3519, 2018.

[16] J. Wang, M. M. Sanchez, Y. Yin, R. Herzer, L. Ma, and O. G. Schmidt, "Silicon-based integrated label-free optofluidic biosensors: Latest advances and roadmap," Advanced Materials Technologies, vol. 5, no. 6, p. $1901138,2020$.

[17] A. M. Jones, C. T. DeRose, A. L. Lentine, A. Starbuck, A. T. Pomerene, and R. A. Norwood, "Racetrack resonator as a loss measurement platform for photonic components," Opt. Express, vol. 23, no. 22, pp. 28883-28 895, Nov 2015. [Online]. Available: http://www.opticsexpress.org/abstract.cfm?URI=oe-23-22-28883

[18] D.-X. Xu, A. Delâge, R. McKinnon, M. Vachon, R. Ma, J. Lapointe, A. Densmore, P. Cheben, S. Janz, and J. H. Schmid, "Archimedean spiral cavity ring resonators in silicon as ultra-compact optical comb filters," Opt. Express, vol. 18, no. 3, pp. 1937-1945, Feb 2010. [Online]. Available: http://www.opticsexpress.org/abstract.cfm?URI=oe18-3-1937

[19] J. Shang, F. Cheng, M. Dubey, J. M. Kaplan, M. Rawal, X. Jiang, D. S. Newburg, P. A. Sullivan, R. B. Andrade, and D. M. Ratner, "An organophosphonate strategy for functionalizing silicon photonic biosensors," Langmuir, vol. 28, no. 6, pp. 3338-3344, 2012, pMID: 22220731. [Online]. Available: https://doi.org/10.1021/la2043153

[20] D. G. Rabus, C. Sada, and K. Rebner, Optofluidics: Process Analytical Technology. Berlin, Boston: De Gruyter, 03 Dec. 2018. [Online]. Available: https://www.degruyter.com/view/title/529258

[21] C. Schulte-Osseili, M. Kleinert, N. Keil, and R. R. Rosencrantz, "Rapid drop-test for lectin binding with glycopolymer-coated optical ring resonators," Biosensors, vol. 9, no. 1, p. 24, 2019.

[22] S. Mudumba, S. de Alba, R. Romero, C. Cherwien, A. Wu, J. Wang, M. A. Gleeson, M. Iqbal, and R. W. Burlingame, "Photonic ring resonance is a versatile platform for performing multiplex immunoassays in real time," Journal of immunological methods, vol. 448, pp. 34-43, 2017.

[23] A. Yariv, "Critical coupling and its control in optical waveguide-ring resonator systems," IEEE Photonics Technology Letters, vol. 14, no. 4, pp. 483-485, 2002.

[24] D. G. Rabus and C. Sada, Integrated Ring Resonators - A Compendium, 2nd ed. Springer Series in Optical Sciences, 2020.

[25] L. Moldenhauer, T. Lipka, D. Venegas-Rojas, K. Igeta, and H. K. Trieu, "Optofluidic a-si:h-based photonic lab-on-chip with dispersion engineered resonance spectra," IEEE Photonics Technology Letters, vol. 29 , no. 4 , pp. 412-415, 2017.

[26] C. K. Madsen and J. H. Zhao, Optical filter design and analysis. Wiley New York, 1999.

[27] R. Boeck, N. A. F. Jaeger, N. Rouger, and L. Chrostowski, "Series-coupled silicon racetrack resonators and the vernier effect: theory and measurement," Opt. Express, vol. 18, no. 24, pp. 25151-25 157, Nov 2010. [Online]. Available: http://www.opticsexpress.org/abstract.cfm?URI=oe-18-24-25151

[28] B. Troia, A. Z. Khokhar, M. Nedeljkovic, J. S. Penades, V. M. N. Passaro, and G. Z. Mashanovich, "Cascade-coupled racetrack resonators based on the vernier effect in the mid-infrared," Opt. Express, vol. 22, no. 20, pp. 23990-24003, Oct 2014. [Online]. Available: http://www.opticsexpress.org/abstract.cfm?URI=oe-22-20-23990

[29] S. K. Selvaraja, G. Winroth, S. Locorotondo, G. Murdoch, A. Milenin, C. Delvaux, P. Ong, S. Pathak, W. Xie, G. Sterckx et al., "193nm immersion lithography for high-performance silicon photonic circuits," in Optical Microlithography XXVII, vol. 9052. International Society for Optics and Photonics, 2014, p. 90520F.

[30] P. Steglich, C. Mai, and A. Mai, "Silicon-organic hybrid photonic devices in a photonic integrated circuit technology," ECS Journal of Solid State Science and Technology, vol. 8, no. 11, pp. Q217-Q221, 2019.

[31] P. Steglich, S. Bondarenko, C. Mai, M. Paul, M. G. Weller, and A. Mai, "Cmos-compatible silicon photonic sensor for refractive index sensing using local back-side release," IEEE Photonics Technology Letters, vol. 32, no. 19, pp. 1241-1244, 2020.

[32] C. Mai, P. Steglich, M. Fraschke, and A. Mai, "Back-side release of slot waveguides for the integration of functional materials in a silicon photonic technology with a full beol," IEEE Transactions on Components, Packaging and Manufacturing Technology, vol. 10, no. 9, pp. 1569-1574, 2020. 
[33] P. Steglich, A. Mai, and C. Mai, "Photonic sensor chip, packaged photonic sensor device and arrangement," Jun. 25 2020, uS Patent App. $16 / 718,595$

[34] C. Mai, P. Steglich, and A. Mai, "Adjustment of the beol for back side module integration on wafer level in a silicon photonic technology," in MikroSystemTechnik 2019; Congress. VDE, 2019, pp. 1-4.

[35] M. Ye, C. Sun, Y. Yu, Y. Ding, and X. Zhang, "Silicon integrated multi-mode ring resonator," Nanophotonics, 2021. [Online]. Available: https://doi.org/10.1515/nanoph-2020-0556

[36] B. E. Little, J. Foresi, G. Steinmeyer, E. Thoen, S. Chu, H. Haus, E. P. Ippen, L. Kimerling, and W. Greene, "Ultra-compact si-sio 2 microring resonator optical channel dropping filters," IEEE Photonics Technology Letters, vol. 10, no. 4, pp. 549-551, 1998.

[37] A. Spott, Y. Liu, T. Baehr-Jones, R. Ilic, and M. Hochberg, "Silicon waveguides and ring resonators at $5.5 \mathrm{~m}, "$ Applied Physics Letters, vol. 97, no. 21, p. 213501, 2010. [Online]. Available: https://doi.org/10.1063/1.3514234

[38] B. Ouyang, Y. Xing, W. Bogaerts, and J. Caro, "Silicon ring resonators with a free spectral range robust to fabrication variations," Opt. Express, vol. 27, no. 26, pp. 38698-38707, Dec 2019. [Online]. Available: http://www.opticsexpress.org/abstract.cfm?URI=oe-27-26-38698

[39] V. W. L. Ho, Y. Chang, Y. Liu, C. Zhang, Y. Li, R. R. Davidson, B. E. Little, G. Wang, and S. T. Chu, "Optical trapping and manipulating with a silica microring resonator in a self-locked scheme," Micromachines, vol. 11, no. 2, 2020. [Online]. Available: https://www.mdpi.com/2072-666X/11/2/202

[40] L. Chrostowski and M. Hochberg, Silicon photonics design: from devices to systems. Cambridge University Press, 2015.

[41] P. Steglich, C. Villringer, S. Dümecke, Y. P. Michel, M. Casalboni, and S. Schrader, "Silicon-on-insulator slot-waveguide design trade-offs," in 2015 International Conference on Photonics, Optics and Laser Technology (PHOTOPTICS), vol. 2. IEEE, 2015, pp. 47-52

[42] T. Visser, H. Blok, B. Demeulenaere, and D. Lenstra, "Confinement factors and gain in optical amplifiers," IEEE Journal of Quantum Electronics, vol. 33, no. 10, pp. 1763-1766, 1997.

[43] P. Steglich, "Silicon-on-insulator slot waveguides: Theory and applications in electro-optics and optical sensing," in Emerging Waveguide Technology. IntechOpen, 2018, pp. 187-210.

[44] P. Steglich, C. Villringer, S. Pulwer, M. Casalboni, and S. Schrader, "Design optimization of silicon-on-insulator slot-waveguides for electrooptical modulators and biosensors," in Photoptics 2015. Springer, 2016, pp. $173-187$.

[45] D. M. Kita, J. Michon, S. G. Johnson, and J. Hu, "Are slot and subwavelength grating waveguides better than strip waveguides for sensing?" Optica, vol. 5, no. 9, pp. 1046-1054, Sep 2018. [Online]. Available: http://www.osapublishing.org/optica/abstract.cfm?URI=optica-5-91046

[46] P. Steglich, C. Mai, D. Stolarek, S. Lischke, S. Kupijai, C. Villringer, S. Pulwer, F. Heinrich, J. Bauer, S. Meister, D. Knoll, M. Casalboni, and S. Schrader, "Novel ring resonator combining strong field confinement with high optical quality factor," IEEE Photonics Technology Letters, vol. 27, no. 20, pp. 2197-2200, 2015.

[47] D.-X. Xu, A. Densmore, A. Delâge, P. Waldron, R. McKinnon, S. Janz, J. Lapointe, G. Lopinski, T. Mischki, E. Post, P. Cheben, and J. H. Schmid, "Folded cavity soi microring sensors for high sensitivity and real time measurement of biomolecular binding," Opt. Express, vol. 16, no. 19, pp. 15 137-15 148, Sep 2008. [Online]. Available: http://www.opticsexpress.org/abstract.cfm?URI=oe-16-19-15137

[48] T. Taniguchi, A. Hirowatari, T. Ikeda, M. Fukuyama, Y. Amemiya, A. Kuroda, and S. Yokoyama, "Detection of antibody-antigen reaction by silicon nitride slot-ring biosensors using protein g," Optics Communications, vol. 365, pp. 16 - 23, 2016. [Online]. Available: http://www.sciencedirect.com/science/article/pii/S0030401815303230

[49] L. Chrostowski, S. Grist, J. Flueckiger, W. Shi, X. Wang, E. Ouellet, H. Yun, M. Webb, B. Nie, Z. Liang et al., "Silicon photonic resonator sensors and devices," in Laser Resonators, Microresonators, and Beam Control XIV, vol. 8236. International Society for Optics and Photonics, 2012 , p. 823620.

[50] I. nigo Molina-Fernández, J. Leuermann, A. O.-M. nux, J. G. Wangüemert-Pérez, and R. Halir, "Fundamental limit of detection of photonic biosensors with coherent phase read-out," Opt. Express, vol. 27, no. 9, pp. 12616-12629, Apr 2019. [Online]. Available: http://www.opticsexpress.org/abstract.cfm?URI=oe-27-9-12616

[51] K. D. Vos, I. Bartolozzi, E. Schacht, P. Bienstman, and R. Baets, "Silicon-on-insulator microring resonator for sensitive and label-free biosensing," Opt. Express, vol. 15, no. 12, pp. 7610-7615, Jun 2007.
[Online]. Available: http://www.opticsexpress.org/abstract.cfm?URI=oe$15-12-7610$

[52] I. White, H. Zhu, J. Suter, N. M. Hanumegowda, H. Oveys, M. Zourob, and X. Fan, "Refractometric sensors for lab-on-a-chip based on optical ring resonators," Sensors Journal, IEEE, vol. 7, no. 1, pp. 28-35, Jan 2007.

[53] P. Steglich, C. Villringer, S. Pulwer, F. Heinrich, J. Bauer, B. Dietzel, C. Mai, A. Mai, M. Casalboni, and S. Schrader, "Hybrid-waveguide ring resonator for biochemical sensing," IEEE Sensors Journal, vol. 17, no. 15, pp. 4781-4790, Aug 2017.

[54] J. Teng, P. Dumon, W. Bogaerts, H. Zhang, X. Jian, X. Han, M. Zhao, G. Morthier, and R. Baets, "Athermal silicon-on-insulator ring resonators by overlaying a polymer cladding on narrowed waveguides," Opt. Express, vol. 17, no. 17, pp. 14627-14633, Aug 2009. [Online]. Available: http://www.opticsexpress.org/abstract.cfm?URI=oe$17-17-14627$

[55] M.-J. Bauls, R. Puchades, and ngel Maquieira, "Chemical surface modifications for the development of silicon-based labelfree integrated optical (io) biosensors: A review," Analytica Chimica Acta, vol. 777, pp. 1 - 16, 2013. [Online]. Available: http://www.sciencedirect.com/science/article/pii/S0003267013001736

[56] A. V. Orlov, A. G. Burenin, N. G. Massarskaya, A. V. Betin, M. P. Nikitin, and P. I. Nikitin, "Highly reproducible and sensitive detection of mycotoxins by label-free biosensors," Sensors and Actuators B: Chemical, vol. 246, pp. 1080 - 1084, 2017. [Online]. Available: http://www.sciencedirect.com/science/article/pii/S0925400516320330

[57] J.-Y. Byeon, F. T. Limpoco, and R. C. Bailey, "Efficient bioconjugation of protein capture agents to biosensor surfaces using aniline-catalyzed hydrazone ligation," Langmuir, vol. 26, no. 19, pp. 15430-15435, 2010, pMID: 20809595. [Online]. Available: https://doi.org/10.1021/la1021824

[58] G. Gaur, S. Hu, R. L. Mernaugh, I. I. Kravchenko, S. T. Retterer, and S. M. Weiss, "Label-free detection of herceptin using suspended silicon microring resonators," Sensors and Actuators B: Chemical, vol. 275, pp. 394 - 401, 2018. [Online]. Available: http://www.sciencedirect.com/science/article/pii/S0925400518313194

[59] M. Morita, T. Ohmi, E. Hasegawa, M. Kawakami, and M. Ohwada, "Growth of native oxide on a silicon surface," Journal of Applied Physics, vol. 68, no. 3, pp. 1272-1281, 1990. [Online]. Available: https://doi.org/10.1063/1.347181

[60] M. Guarisco, D. Gandolfi, R. Guider, L. Vanzetti, R. Bartali, M. Ghulinyan, M. Cretich, M. Chiari, P. Bettotti, L. Pavesi, C. Pederzolli, and L. Pasquardini, "A new aptamer immobilization strategy for protein recognition," Sensors and Actuators B: Chemical, vol. 252, pp. 222 - 231, 2017. [Online]. Available: http://www.sciencedirect.com/science/article/pii/S0925400517309565

[61] M. Antoniou, D. Tsounidi, P. S. Petrou, K. G. Beltsios, and S. E. Kakabakos, "Functionalization of silicon dioxide and silicon nitride surfaces with aminosilanes for optical biosensing applications," MEDICAL DEVICES \& SENSORS, vol. 3, no. 5, p. e10072, 2020. [Online]. Available: https://onlinelibrary.wiley.com/doi/abs/10.1002/mds3.10072

[62] A. K. Wong and U. J. Krull, "Surface characterization of 3glycidoxypropyltrimethoxysilane films on silicon-based substrates," Analytical and bioanalytical chemistry, vol. 383, no. 2, pp. 187-200, 2005.

[63] M. S. McClellan, L. L. Domier, and R. C. Bailey, "Label-free virus detection using silicon photonic microring resonators," Biosensors and Bioelectronics, vol. 31, no. 1, pp. 388 - 392, 2012. [Online]. Available: http://www.sciencedirect.com/science/article/pii/S0956566311007445

[64] M. K. Park, J. S. Kee, J. Y. Quah, V. Netto, J. Song, Q. Fang, E. M. La Fosse, and G.-Q. Lo, "Label-free aptamer sensor based on silicon microring resonators," Sensors and Actuators B: Chemical, vol. 176, pp. 552 - 559, 2013. [Online]. Available: http://www.sciencedirect.com/science/article/pii/S0925400512008921

[65] T. Komikawa, M. Tanaka, K. Yanai, B. R. Johnson, K. Critchley, T. Onodera, S. D. Evans, K. Toko, and M. Okochi, "A bioinspired peptide matrix for the detection of 2,4,6-trinitrotoluene (tnt)," Biosensors and Bioelectronics, vol. 153, p. 112030, 2020. [Online]. Available: http://www.sciencedirect.com/science/article/pii/S0956566320300270

[66] L. Eisner, I. Wilhelm, G. Flachenecker, J. Hrttlen, and W. Schade, "Molecularly imprinted sol-gel for tnt detection with optical micro-ring resonator sensor chips," Sensors, vol. 19, no. 18, 2019. [Online]. Available: https://www.mdpi.com/1424-8220/19/18/3909

[67] K. Gajos, K. Szafraniec, P. Petrou, and A. Budkowski, "Surface density dependent orientation and immunological recognition of antibody on silicon: Tof-sims and surface analysis of two covalent immobilization methods," Applied 
Surface Science, vol. 518, p. 146269, 2020. [Online]. Available: http://www.sciencedirect.com/science/article/pii/S0169433220310254

[68] B. Schroeder, H. Le Xuan, J. L. Vlzke, and M. G. Weller, "Preactivation crosslinkingan efficient method for the oriented immobilization of antibodies," Methods and Protocols, vol. 2, no. 2, 2019. [Online]. Available: https://www.mdpi.com/2409-9279/2/2/35

[69] M. Paul and M. G. Weller, "Antibody screening by microarray technologydirect identification of selective high-affinity clones," Antibodies, vol. 9, no. 1, 2020. [Online]. Available: https://www.mdpi.com/2073-4468/9/1/1

[70] T. Ikeda, Y. Hata, K. ichi Ninomiya, Y. Ikura, K. Takeguchi, S. Aoyagi, R. Hirota, and A. Kuroda, "Oriented immobilization of antibodies on a silicon wafer using si-tagged protein a," Analytical Biochemistry, vol. 385, no. 1, pp. 132 - 137, 2009. [Online]. Available: http://www.sciencedirect.com/science/article/pii/S0003269708007409

[71] D. Vermeulen, S. Selvaraja, P. Verheyen, G. Lepage, W. Bogaerts, P. Absil, D. Van Thourhout, and G. Roelkens, "High-efficiency fiberto-chip grating couplers realized using an advanced cmos-compatible silicon-on-insulator platform," Optics express, vol. 18, no. 17, pp. 18278-18 283, 2010.

[72] D. Taillaert, W. Bogaerts, P. Bienstman, T. F. Krauss, P. Van Daele, I. Moerman, S. Verstuyft, K. De Mesel, and R. Baets, "An outof-plane grating coupler for efficient butt-coupling between compact planar waveguides and single-mode fibers," IEEE Journal of Quantum Electronics, vol. 38, no. 7, pp. 949-955, 2002.

[73] G. Son, S. Han, J. Park, K. Kwon, and K. Yu, "High-efficiency broadband light coupling between optical fibers and photonic integrated circuits," Nanophotonics, vol. 7, no. 12, pp. 1845-1864, 2018.

[74] A. Mekis, S. Gloeckner, G. Masini, A. Narasimha, T. Pinguet, S. Sahni, and P. De Dobbelaere, "A grating-coupler-enabled cmos photonics platform," IEEE Journal of Selected Topics in Quantum Electronics, vol. 17, no. 3, pp. 597-608, 2010.

[75] R. Marchetti, C. Lacava, L. Carroll, K. Gradkowski, and P. Minzioni, "Coupling strategies for silicon photonics integrated chips," Photonics Research, vol. 7, no. 2, pp. 201-239, 2019.

[76] L. M. Weituschat, W. Dickmann, J. Guimbao, D. Ramos, S. Kroker, and P. A. Postigo, "Photonic and thermal modelling of microrings in silicon, diamond and gan for temperature sensing," Nanomaterials, vol. 10, no. 5, p. $934,2020$.

[77] A. Mai, S. Bondarenko, C. Mai, and P. Steglich, "Photonic thermal sensor integration towards electronic-photonic-ic technologies," in ESSDERC 2019 - 49th European Solid-State Device Research Conference (ESSDERC), 2019, pp. 254-257.

[78] P. Steglich, M. Paul, C. Mai, A. Böhme, S. Bondarenko, M. G. Weller and A. Mai, "A monolithically integrated micro fluidic channel in a silicon-based photonic-integrated-circuit technology for biochemical sensing," in Proc. SPIE 11772, Optical Sensors 2021, 2021.

[79] H. Su and X. G. Huang, "Fresnel-reflection-based fiber sensor for online measurement of solute concentration in solutions," Sensors and Actuators B: Chemical, vol. 126, no. 2, pp. 579-582, 2007.

[80] X. Tu, J. Song, T.-Y. Liow, M. K. Park, J. Q. Yiying, J. S. Kee, M. Yu, and G.-Q. Lo, "Thermal independent silicon-nitride slot waveguide biosensor with high sensitivity," Opt. Express, vol. 20, no. 3, pp. 2640-2648, Jan 2012. [Online]. Available: http://www.opticsexpress.org/abstract.cfm?URI=oe-20-3-2640 